\title{
Precise Higgs-background predictions: merging NLO QCD and squared quark-loop corrections to
} four-lepton $+0,1$ jet production

\author{
F. Cascioli, ${ }^{a}$ S. Höche, ${ }^{b}$ F. Krauss, ${ }^{c}$ P. Maierhöfer, ${ }^{a}$ S. Pozzorini ${ }^{a}$ and F. Siegert ${ }^{d}$ \\ ${ }^{a}$ Institut für Theoretische Physik, Universität Zürich, \\ 8057 Zürich, Switzerland \\ ${ }^{b}$ SLAC National Accelerator Laboratory, \\ Menlo Park, CA 94025, U.S.A. \\ ${ }^{c}$ Institute for Particle Physics Phenomenology, Durham University, \\ Durham DH1 3LE, U.K. \\ ${ }^{d}$ Physikalisches Institut, Albert-Ludwigs-Universität Freiburg, \\ D-79104 Freiburg, Germany \\ E-mail: cascioli@physik.uzh.ch, shoeche@slac.stanford.edu, \\ frank.krauss@durham.ac.uk, philipp@physik.uzh.ch, \\ pozzorin@physik.uzh.ch, frank.siegert@cern.ch
}

ABSTRACT: We present precise predictions for four-lepton plus jets production at the LHC obtained within the fully automated SHERPA + OpEnLOOPS framework. Off-shell intermediate vector bosons and related interferences are consistently included using the complexmass scheme. Four-lepton plus 0- and 1-jet final states are described at NLO accuracy, and the precision of the simulation is further increased by squared quark-loop NNLO contributions in the gg $\rightarrow 4 \ell$, gg $\rightarrow 4 \ell+\mathrm{g}, \mathrm{g} q \rightarrow 4 \ell+q$, and $q \bar{q} \rightarrow 4 \ell+\mathrm{g}$ channels. These NLO and NNLO contributions are matched to the SHERPA parton shower, and the 0- and 1-jet final states are consistently merged using the MePs@NLO technique. Thanks to Sudakov resummation, the parton shower provides improved predictions and uncertainty estimates for exclusive observables. This is important when jet vetoes or jet bins are used to separate four-lepton final states arising from Higgs decays, diboson production, and top-pair production. Detailed predictions are presented for the ATLAS and CMS $\mathrm{H} \rightarrow \mathrm{WW}^{*}$ analyses at $8 \mathrm{TeV}$ in the 0 - and 1-jet bins. Assessing renormalisation-, factorisation- and resummationscale uncertainties, which reflect also unknown subleading Sudakov logarithms in jet bins, we find that residual perturbative uncertainties are as small as a few percent.

KEywords: NLO Computations, Monte Carlo Simulations

ARXIV EPRINT: 1309.0500 


\section{Contents}

1 Introduction 1

2 NLO, matching and merging with SHERPA and OPENLoOps 5

2.1 Loop amplitudes with OpenLoops and Collier 5

2.2 Matching to parton shower and merging in SHERPA 6

2.3 Merging of squared quark-loop contributions to four-lepton production 9

3 Monte-Carlo simulations $\quad 9$

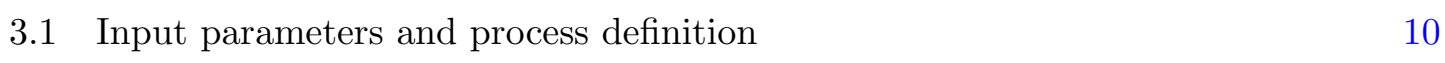

$\begin{array}{lll}3.2 & \text { Fixed-order ingredients of the calculation } & 10\end{array}$

3.3 Matching to the parton shower, multi-jet NLO merging, and scale variations 12

4 Analysis of inclusive $\ell \nu \ell \nu+0,1$ jets production $\quad 15$

$\begin{array}{lll}4.1 & \text { Fixed-order, matched and merged NLO simulations } & 15\end{array}$

$\begin{array}{lll}4.2 \text { Squared quark-loop contributions } & 18\end{array}$

$5 \quad$ Atlas and Cms $\mathbf{H} \rightarrow \mathrm{WW}^{*}$ analyses in the 0- and 1-jet bins 23

5.1 Kinematic distributions after pre-selection cuts 24

5.2 Kinematic distributions in control and signal regions 25

5.3 Exclusive 0- and 1-jet bin cross sections in control and signal regions 29

6 Conclusions $\quad 34$

$\begin{array}{ll}\text { A Treatment of bottom- and top-quark contributions } & 37\end{array}$

B Cuts of the Atlas and CMs $\mathrm{H} \rightarrow \mathrm{WW}^{*}$ analyses in 0- and 1-jet bins 39

\section{Introduction}

Final states involving four leptons played a key role in the discovery of the Higgs boson [1, 2] and will continue to be crucial in the understanding of its properties and coupling structure. There are two classes of final states of interest, namely those consistent with $\mathrm{H} \rightarrow \mathrm{ZZ}^{*}$ decays yielding four charged leptons and those related to $\mathrm{H} \rightarrow \mathrm{WW}^{*}$ resulting in two charged leptons and two neutrinos. They have quite different backgrounds, and for the latter, the dominant and large top-pair production background necessitates the introduction of jet vetoes to render the signal visible. More precisely, four-lepton final states consistent with $\mathrm{H} \rightarrow \mathrm{WW}^{*}$ decays are split into exclusive bins with 0,1 and 2 jets. The separate analysis of the different jet bins permits to disentangle Higgs production 
via gluon fusion from the vector-boson fusion (VBF) production mode. In addition, datadriven determinations of the $\mathrm{H} \rightarrow \mathrm{WW}^{*}$ background take advantage of the fact that its two leading components - diboson and top-pair production - deliver final states of different jet multiplicity. While diboson production represents the leading background in the 0-jet bin, the top-production component becomes slightly more important in the 1-jet bin and clearly dominant in the 2-jet bin.

Due to the absence of a mass peak and the high background cross section, the experimental analyses suffer from signal-to-background ratios as low as around 10 percent. It is thus clear that the precision of the employed background-determination techniques, and the related error estimates, play a crucial role for any Higgs-boson measurement in this channel. In fact, with the statistics available at the end of the LHC run at $8 \mathrm{TeV}$, systematic errors resulting from the background subtraction already dominate the total uncertainty.

In the $\mathrm{H} \rightarrow \mathrm{WW}^{*}$ analyses by ATLAS [3] and CMS [4] a data-driven approach is used to reduce uncertainties in the simulation of the two leading backgrounds. The top-production contribution is fitted to data in a top-enriched control sample. Using Monte-Carlo tools, the top background is extrapolated to the signal region and to an independent dibosonenriched control region. This latter region is used to normalise the diboson background after subtraction of the top contamination. The diboson background is then extrapolated to the signal region using Monte-Carlo predictions. While this approach reduces theoretical uncertainties associated with the background normalisation, the extrapolations between the various control and signal sub-samples rely on Monte-Carlo modelling of the background shapes.

Given that the accuracy of present Higgs-boson measurements requires extrapolation uncertainties at the percent level, it is clear that Monte-Carlo simulations should include all available correction effects and appropriate error estimates. In this context, due to various nontrivial features of the $\mathrm{H} \rightarrow \mathrm{WW}^{*}$ analyses, the requirements in terms of theoretical precision go beyond the mere inclusion of higher-order corrections to inclusive four-lepton production. First, a reliable modelling of the various jets associated to the four-lepton final state requires higher-order QCD corrections up to the highest relevant jet multiplicity. Second, in order to describe potentially large Sudakov logarithms and related uncertainties, which arise from jet vetoes and exclusive jet bins, fixed-order predictions should be matched to parton showers or supplemented by appropriate resummations. Third, vector bosons are produced well below their mass shell in $\mathrm{H} \rightarrow \mathrm{WW}^{*} \rightarrow \ell \nu \ell \nu$ decays. Theoretical predictions for background processes should thus account for corresponding off-shell effects, including non-resonant channels and related interferences.

In this paper we will concentrate on diboson production, which represents about 75 and 40 percent of the $\mathrm{H} \rightarrow \mathrm{WW}^{*} \rightarrow \ell \nu \ell \nu$ background in the 0 - and 1-jet bins, respectively. While we are especially interested in the Higgs-boson analyses, diboson production plays an important role also for precision tests of the Standard Model, vector-boson scattering, searches for anomalous couplings, or as a background in numerous searches.

Higher-order QCD corrections to diboson production at hadron colliders have been extensively studied in the literature. Next-to-leading order (NLO) corrections to inclusive W-pair final states [5-10] amount to roughly $50 \%$ at the LHC and can be further enhanced 
in the tails of distributions or reduced by jet vetoes. Due to the gluon-(anti)quark channels, which start contributing to $\mathrm{pp} \rightarrow \mathrm{W}^{+} \mathrm{W}^{-}$only at NLO, the size of the corrections largely exceeds estimates based on leading-order (LO) scale variations. The matching of NLO predictions for WW production to parton showers was first studied in ref. [11] using the MC@NLO method [11], while the POWHEG matching [12] for WW, WZ and ZZ production, including spin-correlated leptonic decays with non-resonant contributions, was presented in ref. [13]. Similar predictions for ZZ production based on the MC@NLO method can be found in ref. [14].

The NLO corrections to $\mathrm{pp} \rightarrow \mathrm{W}^{+} \mathrm{W}^{-} j$ were presented in refs. [15-17], including spincorrelated leptonic decays and off-shell effects associated with the Breit-Wigner distributions of the resonant W-bosons. At the $14 \mathrm{TeV}$ LHC with rather inclusive cuts the corrections are slightly above $30 \%$. Also in this case, due to the opening of the $g g \rightarrow \mathrm{W}^{+} \mathrm{W}^{-} q \bar{q}$ channel at NLO, the corrections largely exceed LO scale variations. This means that uncertainty estimates based on scale variations start to be meaningful only at NLO. The inclusion of QCD corrections is thus essential in order to improve both, theoretical predictions and error estimates. The matching of NLO pp $\rightarrow \mathrm{W}^{+} \mathrm{W}^{-} j$ calculations to parton showers remains to be addressed in the literature.

Higher-order QCD effects have been studied in quite some detail also for $\mathrm{pp} \rightarrow \mathrm{WW} j j$ in the VBF- and QCD-production modes. In the VBF case, NLO corrections including resonant and non-resonant leptonic decays [18] have been matched to parton showers [19]. For QCD-induced $\mathrm{W}^{+} \mathrm{W}^{-} j j$ production, NLO predictions have been presented by two independent groups, including spin-correlated leptonic decays as Breit-Wigner resonances [20] or in narrow-width approximation [21]. Depending on the scale choice and the collision energy, NLO effects at the LHC can range from a few percent to tens of percent [20]. Up to date, only NLO QCD corrections to same-sign WW $j j$ production $[22,23]$ have been matched to parton showers [24]. Recently, NLO predictions became available also for $\mathrm{pp} \rightarrow W Z j j$ [25].

While full NNLO corrections to diboson production are not yet available, the finite and gauge-invariant contribution from squared quark-loop $\mathrm{gg} \rightarrow \mathrm{W}^{+} \mathrm{W}^{-}$amplitudes was studied in detail in refs. [26-29]. Due to the large gluon flux, such NNLO terms increase the inclusive $\mathrm{W}^{+} \mathrm{W}^{-}$cross section by $3-5 \%$ at the LHC. Their relative importance is known to increase in the $\mathrm{H} \rightarrow \mathrm{WW}^{*}$ analysis. While in presence of tight cuts it can reach up to $30 \%[26,27]$, with the cuts currently applied by the LHC experiments it remains around $10 \%[28,29]$, which corresponds to about half of the Higgs-boson signal. In spite of the tiny Higgs-boson width, the interference of the gg $\rightarrow 4 \ell$ continuum with the signal can reach order $10 \%$ of the gg $\rightarrow 4 \ell$ signal-plus-background cross section [27, 28]. This interference contribution arises almost entirely above threshold, i.e. at invariant masses $M_{\mathrm{WW}}>2 M_{\mathrm{W}}$, and is strongly suppressed at small dilepton invariant mass as well as in the transversemass region $m_{\mathrm{T}} \lesssim M_{\mathrm{H}}[28,30]$. In ref. [29] it was shown that also $\mathrm{pp} \rightarrow \mathrm{W}^{+} \mathrm{W}^{-} j$ receives a significant $\mathrm{gg} \rightarrow \mathrm{W}^{+} \mathrm{W}^{-}$g contribution from squared quark-loop amplitudes, which can reach 6-9\% when Higgs-search cuts are applied.

In this paper we present new precise predictions for four-lepton plus 0- and 1-jet production, ${ }^{1}$ obtained within the fully automated SHERPA+OPEnLoops framework [32,

\footnotetext{
${ }^{1}$ First partial results of this study were anticipated in [31].
} 
33]. The OpenLoops [33] algorithm is an automated generator of virtual QCD corrections to Standard-Model processes, which uses the ColLIER library [34] for the numerically stable evaluation of tensor integrals [35, 36] and scalar integrals [37]. Thanks to a fully flexible interface of SHERPA with OPEnLOOPS, the entire generation chain - from process definition to collider observables - is fully automated and can be steered through SHERPA run cards.

The simulation presented in this paper is the first phenomenological application of Sherpa+OpenLoops. It comprises all previously known QCD contributions to pp $\rightarrow$ $4 \ell$ and $\mathrm{pp} \rightarrow 4 \ell+1 j$, and extends them in various respects. For both processes, NLO corrections are matched to the SHERPA parton shower [32] using the fully colour-correct formulation [38, 39] of the Mc@NLO method [11]. ${ }^{2}$ Using the recently developed multi-jet merging at NLO $[40,41]$, the two MC@NLO samples are consistently merged in a single simulation, which preserves the logarithmic accuracy of the shower and simultaneously guarantees NLO accuracy in the 0- and 1-jet bins. Also squared quark-loop contributions to $\mathrm{pp} \rightarrow 4 \ell+0,1$ jets are included. In addition to the pure gluonic channels, $\mathrm{gg} \rightarrow 4 \ell$ and $\mathrm{gg} \rightarrow 4 \ell+\mathrm{g}$, also the quark-induced $q g \rightarrow 4 \ell+q, \bar{q} g \rightarrow 4 \ell+\bar{q}$, and $q \bar{q} \rightarrow 4 \ell+\mathrm{g}$ channels are taken into account. Moreover, the various squared quark-loop contributions are matched to the parton shower and merged in a single sample. To guarantee an exact treatment of spin correlations and off-shell vector bosons, the complex-mass scheme [42] is used, and all resonant and non-resonant four-lepton plus jets topologies are taken into account.

Detailed predictions are presented for the case of $\mathrm{W}$-pair plus jets production as a signal, as well as for the irreducible background to the AtLAs and CMS H $\rightarrow \mathrm{WW}^{*}$ analyses in the 0 - and 1-jet bins. To illustrate the relative importance of the various contributions, merged NLO predictions are contrasted with an inclusive MC@NLO simulation of pp $\rightarrow 4 \ell$, with separate NLO results for four-lepton plus 0- and 1-jet production, and with squared quark-loop contributions. Residual perturbative uncertainties are assessed by means of scale variations. In addition to the usual renormalisation- and factorisation-scale variations, also the resummation scale of the SHERPA parton shower is varied. This reflects subleading Sudakov logarithms beyond the shower approximation, which renders error estimates more realistic in presence of jet vetoes.

The presented simulation involves various interesting improvements for the $\mathrm{H} \rightarrow \mathrm{WW}^{*}$ analyses. The NLO matching and merging of $\mathrm{pp} \rightarrow 4 \ell+0,1$ jets provides NLO accurate predictions and Sudakov resummation in the first two exclusive jet bins. The inclusion of pp $\rightarrow 4 \ell+1 j$ at NLO, which contributes, as a result of merging, both to the 0 - and 1-jet bins, guarantees that all $q \bar{q}, q g, \bar{q} g$ and gg channels are open. In this situation scale variations can be regarded as more realistic estimates of theoretical uncertainties. Matching and merging render squared quark-loop gg $\rightarrow 4 \ell$ contributions to exclusive jet bins more reliable. In fact, if not supplemented by shower emissions, the parton-level $g g \rightarrow 4 \ell$ channel completely misses the Sudakov suppression induced by the jet veto. Matching gg $\rightarrow 4 \ell$ to the parton shower automatically implies fermion-loop processes with initial-state quarks,

\footnotetext{
${ }^{2}$ In the following, MC@NLO refers to the algorithm of refs. [38, 39], which is an extension of the original MC@NLO method by Frixione and Webber [11]. In particular, we never refer to the Mc@NLO event generator.
} 
like $q \mathrm{~g} \rightarrow 4 \ell+q$, which result from $q \rightarrow q \mathrm{~g}$ shower splittings. The corresponding quarkinduced matrix elements, which are included for the first time in this study, provide an improved description of hard jet emission.

Finally we point out that, while the presented simulation deals only with $\mu^{+} \nu_{\mu} \mathrm{e}^{-} \bar{\nu}_{\mathrm{e}}+$ jets final states, the employed tools allow for a fully automated generation of any other combination of charged leptons and neutrinos.

The paper is organised as follows. In section 2 we discuss the calculation of one-loop amplitudes with OpenLoops and Collier as well as NLO matching and merging in SHERPA. Details of the Monte-Carlo simulations can be found in section 3. In section 4 we present results for inclusive WW-signal cuts, with emphasis on squared quark-loop contributions, merging aspects and jet-veto effects. Section 5 is devoted to a detailed discussion of the $\mathrm{H} \rightarrow \mathrm{WW}^{*}$ analyses at the LHC. Our conclusions are presented in section 6 . Appendix A describes the treatment of bottom- and top-quark contributions, and the $\mathrm{H} \rightarrow \mathrm{WW}^{*}$ selection cuts are documented in appendix B.

\section{NLO, matching and merging with SHERPA and OPENLoops}

This section is devoted to the automation of NLO calculations in SHERPA+OPENLoops and to methodological aspects of matching and merging of NLO and squared quark-loop corrections.

\subsection{Loop amplitudes with OpenLoops and Collier}

For the calculation of virtual corrections we employ OpenLoops [33], a fully automated generator of Standard-Model scattering amplitudes at one loop. The OpenLoops method has been designed in order to break various bottlenecks in multi-particle one-loop calculations. The algorithm is formulated in terms of Feynman diagrams and tensor integrals, which allows for very high CPU efficiency to be achieved. While this was already known from $2 \rightarrow 4$ NLO calculations based on algebraic methods [43-46], the idea behind OpenLOOPs is to replace algebraic manipulations of Feynman diagrams by a numerical recursion, which results in order-of-magnitude reductions both in the size of the numerical code and in the time needed to generate it. Thanks to these improvements, which are accompanied by a further speedup of loop amplitudes at runtime, OpenLoops is able to address largescale problems, such as NLO simulations for classes of processes involving a large number of multi-leg partonic channels.

The OpenLoops recursion is based on the well known idea that one-loop Feynman diagrams can be cut-opened in such a way that the resulting tree-like objects can be generated with automated tree algorithms. However, rather than relying on conventional tree algorithms, the recursion is formulated in terms of loop-momentum polynomials called "open loops". An analogous idea was proposed in ref. [47] in the framework of DysonSchwinger off-shell recursions. Diagrams involving $N$ loop propagators are built by reusing components from related diagrams with $N-1$ loop propagators in a systematic way. Together with other techniques to speed up colour and helicity summations [33], this allows to handle multi-particle processes with up to $\mathcal{O}\left(10^{4}-10^{5}\right)$ one-loop diagrams. 
The algorithm is completely general, since the kernel of the reduction depends only on the Feynman rules of the model at hand, and once implemented it is applicable to any process. Similarly, the so-called $R_{2}$ rational terms [48] are generated as counterterm-like diagrams from corresponding Feynman rules.

For the numerical evaluation of one-loop tensor integrals, OpenLoops is interfaced to the Collier library [34], which implements the Denner-Dittmaier reduction methods $[35,36]$ and the scalar integrals of ref. [37]. Thanks to a variety of expansions in Gram determinants and other kinematic quantities [36], the ColLIER library systematically avoids spurious singularities in exceptional phase-space regions. This allows for a fast and numerically stable evaluation of tensor integrals in double precision. Alternatively, OPP reduction [49] can be used instead of tensor integrals.

The present implementation of OpenLoops can handle one-loop QCD corrections to any Standard-Model process with up to six particles attached to the loops. ${ }^{3}$ Virtual QCD corrections are computed exactly, and the full set of Feynman diagrams contributing to a given process is taken into account by default. For final states involving four leptons, the complex-mass scheme [42] is used for a consistent description of resonant and nonresonant vector-boson propagators and their interferences. OpenLoops can also be used to compute squared one-loop matrix elements, such as the various squared quark-loop amplitudes considered in this paper. The correctness of one-loop amplitudes generated with OpenLoops has been tested systematically against an independent in-house generator for more than one hundred different parton-level processes, and agreement at the level of 12-14 digits on average was found. The first public version of the code will be released in the course of 2013.

\subsection{Matching to parton shower and merging in SHERPA}

The combination of fixed-order calculations and resummation is essential for the analysis of exclusive cross sections. Parton showers implement resummation in a simple, yet effective way. While formally only correct to leading-logarithmic accuracy, they include a number of features that are important for a realistic prediction of exclusive jet spectra. Firstly, the strong coupling factors associated to quark and gluon emissions are evaluated at scales set by the transverse momenta in the parton branchings. This choice sums higher-logarithmic corrections, originating in the enhanced probability for soft and collinear radiation. Secondly, modern parton showers naturally implement local four-momentum conservation in each individual parton emission, which leads to a realistic description of the kinematics in multi-particle final states. Thirdly, most parton showers include higher-logarithmic corrections in an effective approximation known as angular ordering. This method yields the correct jet rates in $\mathrm{e}^{+} \mathrm{e}^{-}$annihilation to hadrons [50], as well as the production of Drell-Yan lepton pairs in hadronic collisions [51].

Cross sections in jet bins as analysed here are strongly sensitive to real radiative corrections, or their suppression. Such corrections are dominated by Sudakov double logarithms

\footnotetext{
${ }^{3}$ Final-state lepton pairs couple to QCD loops only via electroweak vector bosons and should thus be counted as a single particle.
} 
of the jet-veto scale, which can have a large impact both on exclusive cross sections and related uncertainty estimates. A priori it is not clear if renormalisation- and factorisationscale variations provide a meaningful estimate of NLO cross sections in jet bins. In fact conventional scale variations can turn out to be artificially small as a consequence of accidental cancellations between Sudakov-enhanced logarithms and contributions that do not depend on the jet veto [52]. In this respect, fixed-order calculations matched to a parton shower allow for more reliable predictions and error estimates. In particular, factorisationand renormalisation-scale uncertainties can be supplemented by independent variations of the resummation scale, i.e. the scale that enters Sudakov logarithms and corresponds to the starting point of the parton-shower evolution. Resummation-scale variations reflect the uncertainties associated with subleading Sudakov logarithms beyond the shower approximation, and independent variations of the factorisation, renormalisation and resummation scales provide a more reliable assessment of theoretical errors in presence of jet bins.

The parton shower used for our calculation is based on Catani-Seymour dipole subtraction [53]. It was described in detail in refs. [54, 55]. Splitting kernels are given by the spin-averaged dipole-insertion operators, taken in the large- $N_{\mathrm{c}}$ limit. The momentum mapping in branching processes is defined by inversion of the kinematics in the dipolesubtraction scheme. The parameters of the parton shower are given by its infrared cutoff, by the resummation scale, and by the precise scale at which the strong coupling is evaluated. This latter scale must be proportional to the transverse momentum $k_{\mathrm{T}}$ in the splitting process, but it may be varied using a prefactor, $b$, of order one. In practice, the explicit form of $k_{\mathrm{T}}$ is dictated by the dipole kinematics, and different prefactors are used for final-state and initial-state evolution. The resummation scale can be chosen freely in principle, but at leading order it must be equal to the factorization scale.

The matching of NLO calculations and parton showers in the MC@NLO method [11] is based on the idea that $\mathcal{O}\left(\alpha_{s}\right)$ expansions of the parton shower can provide local subtraction terms (called MC counterterms), which cancel all infrared singularities in real-emission matrix elements. The subtracted result is a finite remainder. When combined with the parton shower it gives the correct $\mathcal{O}\left(\alpha_{s}\right)$ distribution of emissions in the radiative phase space. The total cross section is obtained to NLO accuracy by adding virtual corrections and integrated $\mathrm{MC}$ counterterms to the Born cross section and combining them into a common seed for the parton shower. The matching procedure effectively restricts the role of the parton shower to QCD emissions beyond NLO.

This method needs to be modified in processes with more than three coloured particles at Born level, because of non-factorisable soft-gluon insertions at real-emission level. Spin correlations further complicate the picture. This problem is solved by using a variant of the original MC@NLO technique [38, 39]. Like SHERPA's parton shower itself, this method is based on the dipole-subtraction formalism by Catani and Seymour [53], and it is implemented in SHERPA in a fully automated way. It supplements the parton shower with spin and colour correlations for the first emission and therefore extends it systematically beyond the large- $N_{\mathrm{c}}$ approximation.

We combine Mc@NLO calculations of varying jet multiplicity into inclusive event samples using the MePs@NLO method [40, 41]. This technique is based on partitioning the 
phase space associated to QCD emissions into a soft and a hard regime. The soft region is filled by the parton shower alone, while the hard region is described in terms of fixed-order calculations, to which the parton shower has been matched. In case of the Mc@NLO simulation with the highest jet multiplicity, $N_{\max }$, the parton shower is allowed to fill the entire phase space. The phase-space separation is achieved in terms of a kinematical variable analogous to the jet criterion in longitudinally-invariant $k_{\mathrm{T}}$-clustering algorithms [56]. We will denote the separation cut by $Q_{\text {cut }}$. It should be chosen smaller than the minimum jet transverse momentum. In this manner, the prediction for inter-jet correlations involving up to $N_{\max }$ jets is always NLO accurate, and augmented by resummation as implemented in the parton shower.

The choice of the renormalisation scale in the MEPS@NLO approach is based on the CKKW technique, a multi-jet merging algorithm for tree-level matrix elements [57]. Each shower emission is associated with a factor $\alpha_{s}\left(b k_{\mathrm{T}}^{2}\right)$, where the scale is dictated by the resummation. The smooth transition between parton-shower and matrix-element regimes at the merging scale $Q_{\text {cut }}$ requires a similar scale choice also in matrix elements. To this end, multi-jet events are clustered into a $2 \rightarrow 2$ core process. The clustering algorithm is defined as an exact inversion of the parton shower, such that clusterings are determined according to the parton-shower branching probabilities [56]. The coupling factors resulting from the various QCD emissions are then evaluated at scales $\mu^{2}=b k_{\mathrm{T}}^{2}$, where $k_{\mathrm{T}}$ is the nodal scale of the corresponding branching, while the $\alpha_{s}^{K}\left(\mu^{2}\right)$ term associated with the core process is taken at the usual scale $\mu=\mu_{\mathrm{R}}$. This latter can be chosen freely as in fixed-order calculations.

In practice, in the MEPS@NLO algorithm all $\alpha_{s}$ terms are first evaluated at the scale $\mu_{\mathrm{R}}$, and the CKKW prescription is implemented via weight-correction factors,

$$
\frac{\alpha_{s}\left(b k_{\mathrm{T}}^{2}\right)}{\alpha_{s}\left(\mu_{\mathrm{R}}^{2}\right)} \approx 1-\frac{\alpha_{s}\left(\mu_{\mathrm{R}}^{2}\right)}{2 \pi} b_{0} \ln \left(\frac{b k_{\mathrm{T}}^{2}}{\mu_{\mathrm{R}}^{2}}\right)
$$

for each branching. More precisely, in LO and NLO matrix elements the left- and righthand sides of (2.1) are used, respectively. For the hard remainder function in the MC@NLO calculations contributing to the MEPS@NLO result the renormalisation scale is always evaluated according to the most likely underlying Born configuration, classified according to the branching probability in the parton shower.

The fact that the CKKW scale choice adapts to the jet kinematics can improve the description of jet emission also at high transverse momentum. In this region, where jet emission is typically associated to CKKW coupling factors $\alpha_{s}\left(p_{\mathrm{T}}^{2}\right)$, fixed-order calculations based on a global renormalisation scale $\mu_{\mathrm{R}}$ involve a relative factor $\alpha_{s}\left(\mu_{\mathrm{R}}^{2}\right) / \alpha_{s}\left(p_{\mathrm{T}}^{2}\right)$, which can significantly overestimate the jet rate if $\mu_{\mathrm{R}}$ does not adapt to the jet transverse momentum and $p_{\mathrm{T}} \gg \mu_{\mathrm{R}}$. This factor tends to be compensated by NLO corrections, but in MC@NLO simulations with fixed jet multiplicity $N$ it remains uncompensated for the $(N+1)$-th jet, whose description relies on real-emission LO matrix elements. Within Meps@Nlo, if $N<N_{\max }$ such real-emission matrix elements are confined at transverse momenta below the merging scale and replaced by an MC@NLO simulation with $N+1$ jets above $Q_{\text {cut }}$. In this way NLO accuracy is ensured for the first $N_{\max }$ jets, and the 
problem remains present only for the subsequent jet. A simple solution consists of including $\left(N_{\max }+1\right)$-jet LO matrix elements in the merging procedure. In this way, also the $\left(N_{\max }+1\right)$-th jet receives a CKKW coupling factor $\alpha_{s}\left(p_{\mathrm{T}}^{2}\right)$ above the merging scale. As discussed in section 3.3, for the MePs@NLO simulation of pp $\rightarrow 4 \ell+0,1 j$ we adopt a dynamical scale $\mu_{\mathrm{R}}$ that depends only on the W-boson transverse energy and does not adapt to extra jet emissions. The above discussion is thus relevant for the high- $p_{\mathrm{T}}$ tail of the second jet, where it's likely that $\mu_{\mathrm{R}} \ll p_{\mathrm{T}}$, since the two jets typically recoil against each other and the transverse energy of the $\mathrm{W}$ bosons tends to remain of the order of $M_{\mathrm{W}}$.

In order to guarantee a complete treatment of scale uncertainties, renormalisation-scale variations in the MEPS@NLO merging approach are performed simultaneously in the fixedorder calculation and in the parton shower. The same rescaling factors are applied to the CKKW scales and to the scale $\mu_{\mathrm{R}}$ used in the $\alpha_{s}$ terms associated with the core process.

\subsection{Merging of squared quark-loop contributions to four-lepton production}

We present here, for the first time, a combination of the squared quark-loop contributions to $\mathrm{pp} \rightarrow 4 \ell+0,1 j$ using the ME+PS merging technique of ref. [56]. At matrix-element level we consider all squared one-loop amplitudes that involve a closed quark loop. While squared quark-loop corrections to $4 \ell$ final states involve only gg initial states, $4 \ell+1 j$ production involves, in addition to $\mathrm{gg} \rightarrow 4 \ell+\mathrm{g}$, also $q \mathrm{~g} \rightarrow 4 \ell+q, \bar{q} \mathrm{~g} \rightarrow 4 \ell+\bar{q}$ and $q \bar{q} \rightarrow 4 \ell+\mathrm{g}$ contributions. For these quark-initiated channels we require that all finalstate leptons are connected to the quark loop via vector-boson exchange, i.e. we exclude topologies where vector bosons couple to the external quark line. The inclusion of these quark-initiated channels is mandatory for a consistent merging of the $4 \ell+0,1 j$ samples. This is due to the fact that gluon- and quark-initiated channels are intimately connected via $q \rightarrow q \mathrm{~g}$ and $\mathrm{g} \rightarrow q \bar{q}$ parton-shower splittings. Including the $q \mathrm{~g}$ and $\bar{q} \mathrm{~g}$ channels ensures that all splitting functions used in the shower are replaced by matrix elements in the hardjet region. The finite contribution from the $q \bar{q} \rightarrow 4 \ell+\mathrm{g}$ channel is added for consistency. While the gg-induced channels have already been discussed in the literature [26-29, 58], the squared quark-loop contributions to the $q \mathrm{~g}-, \bar{q} \mathrm{~g}$ - and $q \bar{q}$-channels are investigated for the first time in this paper.

To merge the $4 \ell+0,1 j$ final states we can use the tree-level techniques of ref. [56] since all involved matrix elements are infrared and ultraviolet finite. In particular, the merging scale $Q_{\text {cut }}$ acts as an infrared cutoff that avoids soft and collinear divergences of $4 \ell+1 j$ matrix elements, and the phase-space region below $Q_{\text {cut }}$ is filled by gg $\rightarrow 4 \ell$ matrix elements plus shower emissions. As discussed in section 3.3, while squared quark-loop corrections represent NNLO contributions to inclusive $4 \ell+0,1 j$ production, their intrinsic accuracy is only leading order. Consequently, as we will see in sections $4-5$, squared quark-loop terms are more sensitive to renormalisation- and resummation-scale variations as compared to MEPS@NLO predictions.

\section{Monte-Carlo simulations}

In the following we discuss input parameters and theoretical ingredients of the Monte-Carlo simulations presented in sections 4 and 5 . 


\subsection{Input parameters and process definition}

The presented results refer to pp $\rightarrow \mu^{+} \nu_{\mu} \mathrm{e}^{-} \bar{\nu}_{\mathrm{e}}+X$ at a centre-of-mass energy of $8 \mathrm{TeV}$. Predictions at NLO and squared quark-loop corrections are evaluated using the five-flavour CT10 NLO parton distributions [59] with the respective running strong coupling $\alpha_{s}$. At LO we employ the CT09MCS PDF set. For the vector-boson masses we use

$$
M_{\mathrm{W}}=80.399 \mathrm{GeV}, \quad M_{\mathrm{Z}}=91.1876 \mathrm{GeV},
$$

and in order to guarantee NLO accurate $\mathrm{W} \rightarrow \ell \nu$ branching fractions we use NLO input widths

$$
\Gamma_{\mathrm{W}}=2.0997 \mathrm{GeV}, \quad \Gamma_{\mathrm{Z}}=2.5097 \mathrm{GeV} .
$$

The electroweak mixing angle is obtained from the ratio of the complex W- and Z-boson masses as [42]

$$
\cos ^{2} \theta_{\mathrm{w}}=\frac{M_{\mathrm{W}}^{2}-\mathrm{i} \Gamma_{\mathrm{W}} M_{\mathrm{W}}}{M_{\mathrm{Z}}^{2}-\mathrm{i} \Gamma_{\mathrm{Z}} M_{\mathrm{Z}}}
$$

and the electromagnetic fine-structure constant is derived from the Fermi constant $G_{\mu}=$ $1.16637 \cdot 10^{-5} \mathrm{GeV}^{-2}$ in the so-called $G_{\mu}$-scheme, which results in

$$
\alpha^{-1}=\frac{\pi}{\sqrt{2} G_{\mu} M_{\mathrm{W}}^{2}}\left(1-\frac{M_{\mathrm{W}}^{2}}{M_{\mathrm{Z}}^{2}}\right)^{-1}=132.348905 .
$$

Since quark-mixing effects cancel almost completely [17], we set the CKM matrix equal to one.

Partonic channels with initial- and final-state b quarks are not included in order to avoid any overlap with $\mathrm{t} \overline{\mathrm{t}}$ and $\mathrm{tW}$ production. At NLO this separation is nontrivial since $\mathrm{W}^{+} \mathrm{W}^{-}+1 j$ production receives $\mathrm{pp} \rightarrow \mathrm{W}^{+} \mathrm{W}^{-} \mathrm{b} \overline{\mathrm{b}}$ real-emission contributions that involve top-quark resonances. At the same time, $\mathrm{W}^{+} \mathrm{W}^{-} \mathrm{b} \overline{\mathrm{b}}$ final states are intimately connected to the virtual corrections to $q \bar{q} \rightarrow \mathrm{W}^{+} \mathrm{W}^{-}$g via cancellations of collinear singularities that arise from $\mathrm{g} \rightarrow \mathrm{b} \overline{\mathrm{b}}$ splittings [17]. This is discussed in detail in appendix A, where we introduce a prescription to separate $\mathrm{W}^{+} \mathrm{W}^{-}+$jets from single-top and top-pair production processes in such a way that each contribution is infrared finite and free from large logarithms associated to $\mathrm{g} \rightarrow \mathrm{b} \bar{b}$ splittings. This prescription is not unique, and we estimate the related ambiguity to be of order $1 \%$. It can be eliminated by a consistent matching of $\mathrm{W}^{+} \mathrm{W}^{-}+$jets and $\mathrm{W}^{+} \mathrm{W}^{-} \mathrm{b} \overline{\mathrm{b}}$ production as explained in appendix $\mathrm{A}$.

\subsection{Fixed-order ingredients of the calculation}

Sample Feynman diagrams contributing to the fixed-order building blocks of the calculation are shown in figures 1 and 2. For brevity $\mu^{+} \nu_{\mu} \mathrm{e}^{-} \bar{\nu}_{\mathrm{e}}$ configurations are often denoted as $\ell \nu \ell \nu$ or $4 \ell$ final states in the following. The first figure illustrates NLO QCD corrections to $\mathrm{pp} \rightarrow 4 \ell$ and $\mathrm{pp} \rightarrow 4 \ell+1 j$, which involve various $\bar{q} q, q \mathrm{~g}, \bar{q} \mathrm{~g}$ and gg partonic channels. The complete set of Feynman diagrams and related interferences is taken into account, including single-resonant $\mathrm{Z} / \gamma^{*} \rightarrow \mathrm{e}^{-} \bar{\nu}_{\mathrm{e}} \mathrm{W}^{+}\left(\rightarrow \mu^{+} \nu_{\mu}\right)$ sub-topologies. Pentagons represent the most involved one-loop topologies. 

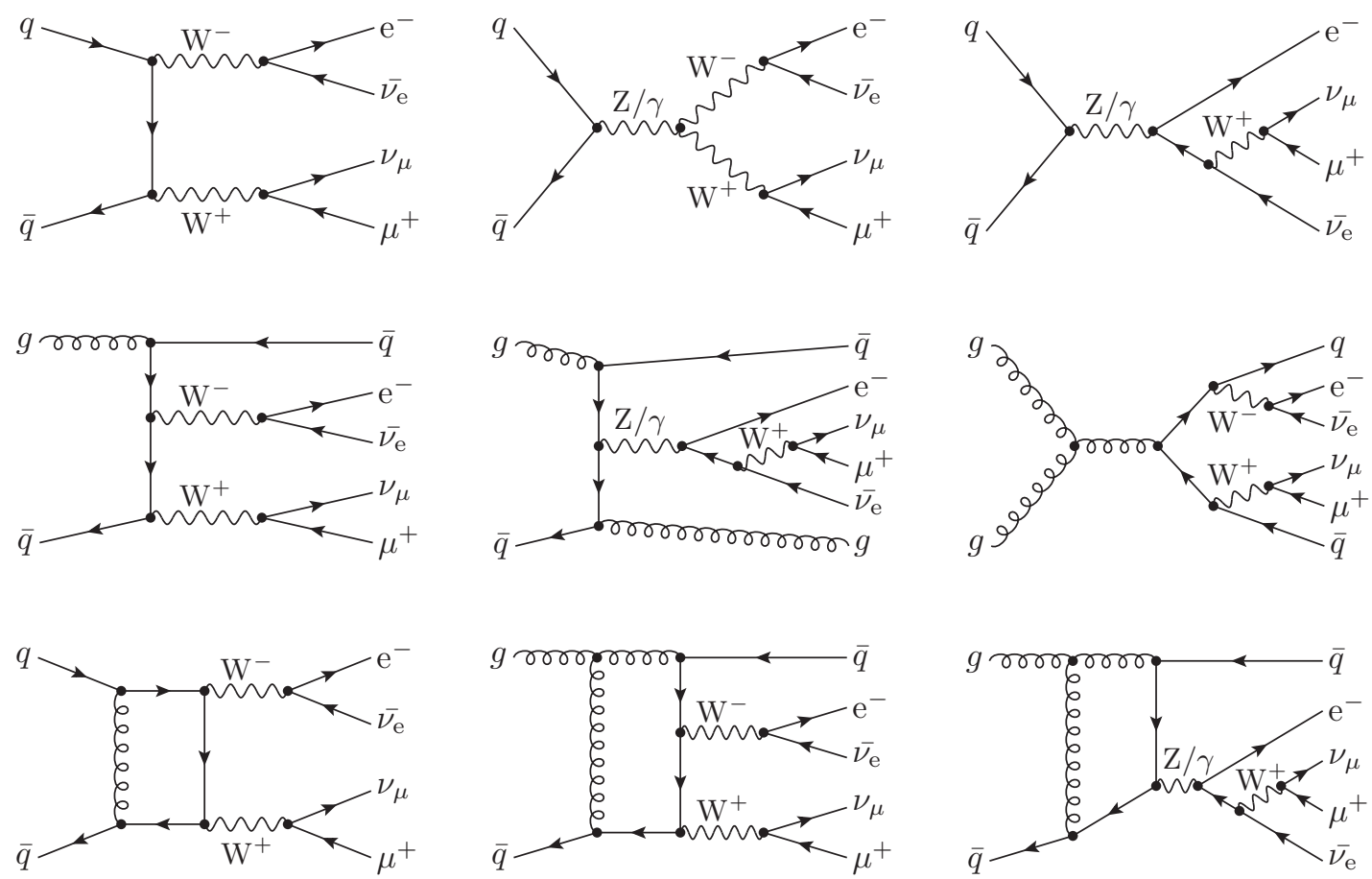

Figure 1. Sample Feynman diagrams contributing to pp $\rightarrow \mu^{+} \nu_{\mu} \mathrm{e}^{-} \bar{\nu}_{\mathrm{e}}$ and $\mathrm{pp} \rightarrow \mu^{+} \nu_{\mu} \mathrm{e}^{-} \bar{\nu}_{\mathrm{e}}+1 j$ at NLO.

In addition to NLO corrections also squared quark-loop contributions to the partonic channels gg $\rightarrow 4 \ell$, gg $\rightarrow 4 \ell+\mathrm{g}, \mathrm{g} q \rightarrow 4 \ell+q, \mathrm{~g} \bar{q} \rightarrow 4 \ell+\bar{q}$, and $q \bar{q} \rightarrow 4 \ell+\mathrm{g}$ are computed. Corresponding Feynman diagrams are shown in figure 2. The most involved diagrams are again pentagons. As discussed in section 2.3, the inclusion of the quark-induced channels is mandatory for a correct description of the full spectrum of jet emission based on the merging of $4 \ell$ and $4 \ell+j$ simulations. Contributions where the leptons are coupled to quark triangles via $Z / \gamma^{*}$ exchange, like in the first two diagrams of figure 2, vanish due to electroweak Ward identities [10]. In contrast, related topologies with an extra gluon in the final state, like the last two diagrams in figure 2, yield non-vanishing contributions. The various NLO and squared quark-loop amplitudes generated for the present study comprise all relevant Higgs-boson contributions, including the interference of the Higgs signal with the four-lepton continuum. However, for the background predictions presented in sections $4-5$ all Higgs-boson contributions have been decoupled by setting $M_{\mathrm{H}} \rightarrow \infty$.

A series of checks has been performed to validate all ingredients of the QCD corrections. To check the correctness of the $q \bar{q} \rightarrow 4 \ell+0,1$ g OpenLoops matrix elements we used an independent computer-algebra generator, originally developed for the calculations of refs. [43, 45]. The squared quark-loop gg $\rightarrow 4 \ell+0,1 \mathrm{~g}$ amplitudes have been checked against MCFM [60] and ref. [29]. The NLO and squared quark-loop integrated cross sections for $\mathrm{pp} \rightarrow 4 \ell+0,1 j$ and $\mathrm{gg} \rightarrow 4 \ell+0,1 \mathrm{~g}$ have been found to agree with various results in the literature $[13,27,29]$. Finally, the NLO cross sections for hadronic $4 \ell+0,1 j$ production have been reproduced with sub-permil statistical precision using an indepen- 

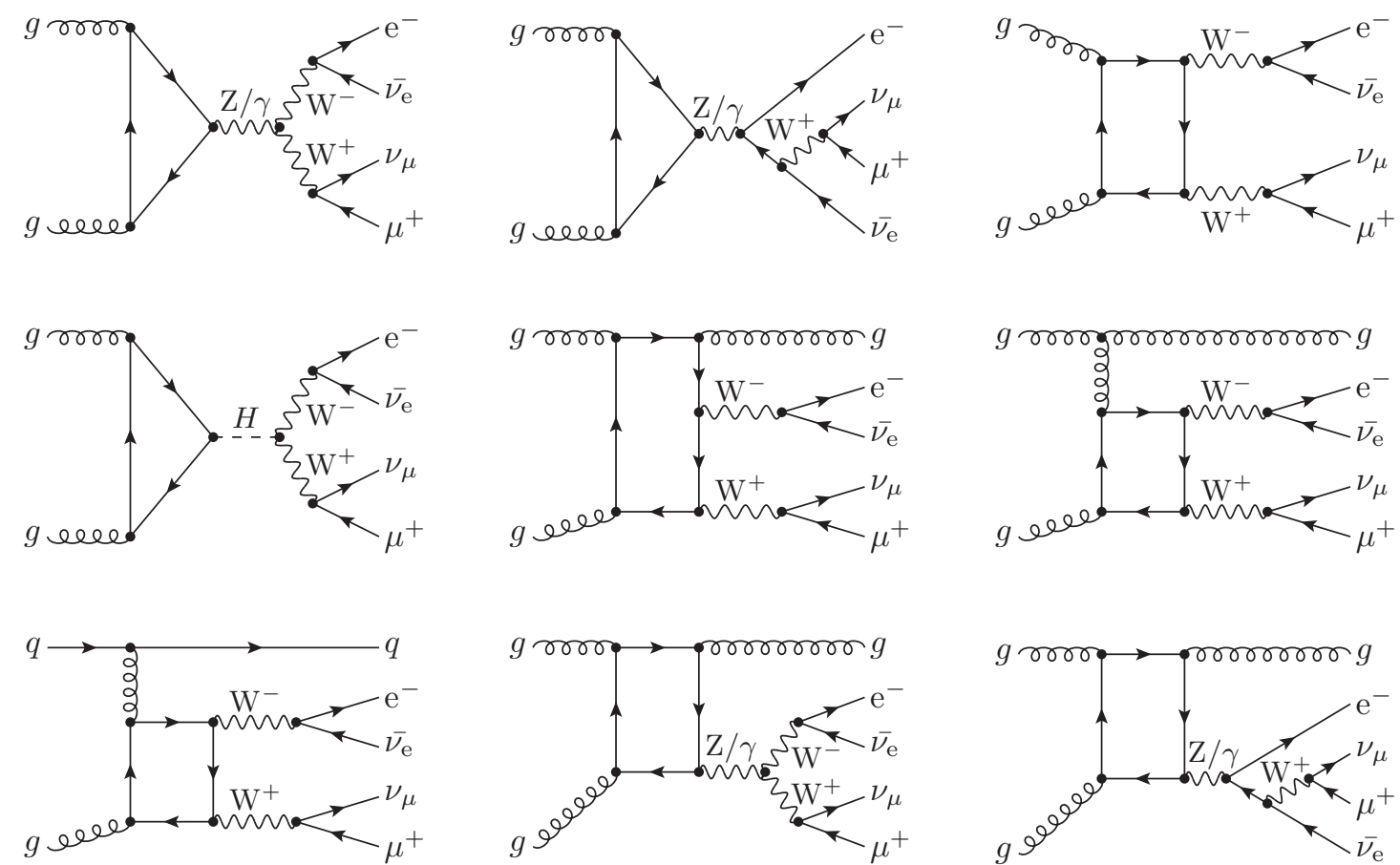

Figure 2. Sample Feynman diagrams involved in the squared quark-loop NNLO contributions to $\mathrm{pp} \rightarrow \mu^{+} \nu_{\mu} \mathrm{e}^{-} \bar{\nu}_{\mathrm{e}}$ and $\mathrm{pp} \rightarrow \mu^{+} \nu_{\mu} \mathrm{e}^{-} \bar{\nu}_{\mathrm{e}}+1 j$.

dent Monte-Carlo generator, which was developed by S. Kallweit in the framework of the $\mathrm{pp} \rightarrow \mathrm{W}^{+} \mathrm{W}^{-} \mathrm{b} \overline{\mathrm{b}}$ calculation of ref. [45].

The calculation of tree-level matrix elements is performed either by the Amegic $++[61]$ or the Comix [62] matrix-element generator, where Comix is used only for $\mathrm{pp} \rightarrow 4 \ell+2 j$ subprocesses. Integrated and real subtraction terms are computed with the method of Catani and Seymour [53], using the automated implementation in AMEGIC $++[63]$.

\subsection{Matching to the parton shower, multi-jet NLO merging, and scale varia- tions}

The perturbative content of the various fixed-order, matched and merged simulations that are presented in sections 4 and 5 is illustrated in table 1 . Parton-level NLO predictions for $\mathrm{pp} \rightarrow 4 \ell+0,1 j$ are denoted as NLO $4 \ell$ and NLO $4 \ell+1 j$. Their NLO predictive power is restricted to the 0 - and 1-jet bins, respectively. ${ }^{4}$ In bins with one extra jet with respect to the simulated process, the precision decreases to LO, and higher-multiplicity bins are not populated at all.

This is overcome by matching NLO $4 \ell$ or NLO $4 \ell+1 j$ matrix elements to the parton shower. Corresponding predictions are denoted as MC@NLO $4 \ell$ and MC@NLO 4 $+1 j$. The

\footnotetext{
${ }^{4}$ In this discussion of the perturbative accuracy we refer to jet bins in the inclusive sense. The 0-, 1and 2-jet bins should namely be understood as final states with $\geq 0, \geq 1$ and $\geq 2$ jets, or equivalently as observables that explicitly or implicitly involve a corresponding number of jets.
} 


\begin{tabular}{|c|c|c|c|}
\hline NLO simulations & 0-jet & 1-jet & 2-jet \\
\hline NLO $4 \ell$ & $\mathrm{NLO}$ & $\mathrm{LO}$ & - \\
\hline $\mathrm{NLO} 4 \ell+1 j$ & - & $\mathrm{NLO}$ & $\mathrm{LO}$ \\
\hline MC@NLO 4l & $\mathrm{NLO}+\mathrm{PS}$ & $\mathrm{LO}+\mathrm{PS}$ & $\overline{P S}$ \\
\hline $\mathrm{MC} @ \mathrm{NLO} 4 \ell+1 j$ & - & $\mathrm{NLO}+\mathrm{PS}$ & $\mathrm{LO}+\mathrm{PS}$ \\
\hline MEPS@NLO $4 \ell+0,1 j$ & $\mathrm{NLO}+\mathrm{PS}$ & $\mathrm{NLO}+\mathrm{PS}$ & $\mathrm{LO}+\mathrm{PS}$ \\
\hline LOOP $^{2}$ simulations & 0-jet & 1-jet & 2-jet \\
\hline $\mathrm{LOOP}^{2} 4 \ell$ & $\mathrm{LO}$ & - & - \\
\hline $\mathrm{LOOP}^{2} 4 \ell+1 j$ & - & $\mathrm{LO}$ & - \\
\hline $\mathrm{LOOP}^{2}+\mathrm{PS} 4 \ell$ & $\mathrm{LO}+\mathrm{PS}$ & $\mathrm{PS}$ & PS \\
\hline $\mathrm{LOOP}^{2}+\mathrm{PS} 4 \ell+1 j$ & 一 & $\mathrm{LO}+\mathrm{PS}$ & PS \\
\hline MEPS@LOOP ${ }^{2} 4 \ell+0,1 j$ & $\mathrm{LO}+\mathrm{PS}$ & $\mathrm{LO}+\mathrm{PS}$ & PS \\
\hline
\end{tabular}

Table 1. Perturbative accuracy of various fixed-order, matched and merged simulations for final states with 0,1 and 2 jets.

shower radiates an arbitrary number of extra jets, which effectively resums large Sudakov logarithms that arise when QCD radiation is constrained by tight cuts, such as in presence of jet vetoes. Similarly as the underlying NLO matrix elements, MC@NLO predictions provide NLO precision only for one particular jet multiplicity. In the following sections we will consider only MC@NLO $4 \ell$ predictions. This corresponds to the usual inclusive NLO+PS samples used in experimental studies, where observables involving one jet are only LO accurate, and the emission of additional jets is entirely based on the parton-shower approximation.

Our best NLO predictions are denoted as MEPS@NLO $4 \ell+0,1 j$ and result from merging MC@NLO $4 \ell$ and MC@NLO $4 \ell+1 j$ samples. This provides shower-improved NLO precision in the first two jet bins. To ensure that the formal NLO accuracy is preserved in the 0 - and 1-jet bins, the merging scale $Q_{\text {cut }}$ should not exceed the $p_{\mathrm{T}}$-threshold used for jet binning. On the other hand, in the limit of small $Q_{\text {cut }}$ the fact that higher-logarithmic terms in the fixed-order NLO $4 \ell+1 j$ calculation are not resummed in the Sudakov form factor gives rise to a logarithmic sensitivity to the merging scale. Such logarithms are beyond the shower accuracy but can be numerically non-negligible [64,65]. Thus the merging scale should not be set too far below the jet- $p_{\mathrm{T}}$ threshold. Following this reasoning the value $Q_{\text {cut }}=20 \mathrm{GeV}$ has been used as merging scale, and the stability of the results with respect to this technical parameter has been tested using variations in the range $15 \mathrm{GeV} \leq Q_{\text {cut }} \leq 35 \mathrm{GeV}$. The corresponding uncertainties are discussed in section 5 for the case of the $\mathrm{H} \rightarrow \mathrm{WW}^{*}$ analysis, where they turn out to be at the percent level. The MePs@NLO $4 \ell+0,1 j$ sample is further improved by including LO matrix elements with two jets in the merging procedure. As explained in section 2.2, this guarantees a better (CKKW-type) scale choice for the $\alpha_{s}$ factor associated with the second jet emission.

In order to gain insights into the importance of parton-shower and merging effects, 
we will present systematic comparisons of NLO, Mc@NLO and MEPS@NLO predictions. While Sudakov resummation effects due to the parton shower show up in the difference between NLO $4 \ell$ and MC@NLO 4 $\ell$, comparing MC@NLO $4 \ell$ to MePs@NLO $4 \ell+0,1 j$ allows one to assess NLO corrections to the first emission.

As already mentioned, squared quark-loop terms included in our simulation represent NNLO contributions to $\mathrm{pp} \rightarrow 4 \ell+(0) 1 j$. On the other hand, since NNLO is the first order at which the gg $\rightarrow 4 \ell+0(1) \mathrm{g}$ channels start contributing to $4 \ell+(0) 1 j$ production, these corrections can also be regarded as LO contributions. As indicated in table 1, squared quark-loop terms behave as LO predictions also for what concerns the number of external QCD partons. In fact, fixed-order squared quark-loop predictions, which we denote as $\operatorname{Loop}^{2} 4 \ell$ and $\operatorname{Loop}^{2} 4 \ell+1 j$, populate only a single jet bin. In particular, $\operatorname{LoOP}^{2} 4 \ell$ predictions completely miss exclusive jet emission and suppression effects resulting from jet vetoes. A first realistic estimate of jet-veto effects is obtained by showering squared quark-loop contributions. The corresponding predictions are labelled as LOOP ${ }^{2}+\mathrm{PS} 4 \ell$ and $\mathrm{LOOP}^{2}+\mathrm{PS} 4 \ell+1 j$, depending on the jet multiplicity of the underlying matrix elements. Merging the $\mathrm{LOOP}^{2}+\mathrm{PS}$ simulations with 0 and 1 jets results in a single MEPs@LoOP ${ }^{2} 4 \ell+$ $0,1 j$ sample, which provides a reliable description of the full spectrum of jet emission, from soft to hard regions. This merged squared quark-loop simulation comprises also partonic channels with initial-state quarks. To assess their relative importance, in section 4, full MEPS@LOOP ${ }^{2} 4 \ell+0,1 j$ predictions are compared to corresponding predictions involving only initial-state gluons.

As a default renormalisation $\left(\mu_{\mathrm{R}}\right)$, factorisation $\left(\mu_{\mathrm{F}}\right)$ and resummation $\left(\mu_{Q}\right)$ scale we adopt the average W-boson transverse energy

$$
\mu_{0}=\frac{1}{2}\left(E_{\mathrm{T}, \mathrm{W}^{+}}+E_{\mathrm{T}, \mathrm{W}^{-}}\right)
$$

where $E_{\mathrm{T}, \mathrm{W}}^{2}=M_{\mathrm{W}}^{2}+\left(\vec{p}_{\mathrm{T}, \ell}+\vec{p}_{\mathrm{T}, \nu}\right)^{2}$. As discussed in section 4, motivated by the comparison of hard-jet emission from parton shower and matrix elements, in the case of squared quarkloop contributions we decided to reduce the resummation scale by a factor two, i.e. we set $\mu_{Q}=\mu_{0} / 2$.

Renormalisation- and factorisation-scale uncertainties are assessed by applying independent variations $\mu_{\mathrm{R}}=\xi_{\mathrm{R}} \mu_{0}$ and $\mu_{\mathrm{F}}=\xi_{\mathrm{F}} \mu_{0}$, with factor-two rescalings $\left(\xi_{\mathrm{R}}, \xi_{\mathrm{F}}\right)=(2,2)$, $(2,1),(1,2),(1,1),(1,0.5),(0.5,1),(0.5,0.5)$. The renormalisation scale is varied in all $\alpha_{s}$ terms that arise in matrix elements or from the shower. In NLO and MC@NLO predictions all $\alpha_{s}$ terms arising from matrix elements are evaluated at $\mu_{\mathrm{R}}=\xi_{\mathrm{R}} \mu_{0}$, while in MEPS@NLO the scale $\mu_{0}$ is used only in tree and loop contributions to the pp $\rightarrow 4 \ell$ core process, which results from $4 \ell+$ jets configurations via clustering of all hard jets. For the $\alpha_{s}$ factors associated with jet emissions a CKKW scale choice is applied, as discussed in section 2.2. As a consequence, MEPS@NLO predictions are less sensitive to the choice of the central scale $\mu_{0}$. Also in MePs@Loop ${ }^{2}$ merging the scale of $\alpha_{s}$ factors associated to QCD emissions is dictated by the CKKW prescription. In this case the core process involves a term $\alpha_{s}^{2}\left(\mu_{\mathrm{R}}\right)$, which renders squared quark-loop corrections more sensitive to the choice of the central scale $\mu_{0}$. 
In addition to usual QCD-scale studies, the SHERPA framework allows also for automated variations of the resummation scale $\mu_{Q}$, which corresponds to the starting scale of the parton shower. This scale is varied by factors $\mu_{Q} / \mu_{0}=1 / \sqrt{2}, 1, \sqrt{2}$, while keeping $\mu_{\mathrm{R}}$ and $\mu_{\mathrm{F}}$ fixed. As discussed in section 2.2, this reflects uncertainties related to subleading logarithms beyond the shower approximation and yields more realistic error estimates for exclusive observables such as jet-vetoed cross sections. In order to quantify the total scale uncertainty we will regard $\left(\mu_{\mathrm{R}}, \mu_{\mathrm{F}}\right)$ and $\mu_{Q}$ variations as uncorrelated and add them in quadrature. ${ }^{5}$ Uncertainties related to the PDFs, $\alpha_{s}\left(M_{\mathrm{Z}}\right)$, hadronisation, and underlying event are not considered in this study.

The presented results were obtained with a SHERPA 2.0 pre-release version. ${ }^{6}$ First partial results of this simulation have been presented in ref. [31]. In addition to the squared quark-loop contributions, which were not included in ref. [31], in this paper we investigate various new observables. Due to the difference between (3.5) and the scale choice $\mu_{0}=M_{\ell \nu \ell \nu}$ in ref. [31], results presented here should not be directly compared to those of ref. [31].

\section{Analysis of inclusive $\ell \nu \ell \nu+0,1$ jets production}

As a first application of our simulation we study $\mu^{+} \nu_{\mu} \mathrm{e}^{-} \bar{\nu}_{\mathrm{e}}$ and $\mu^{+} \nu_{\mu} \mathrm{e}^{-} \bar{\nu}_{\mathrm{e}}+1$ jet production without any Higgs-analysis specific cuts. To this end we adopt the cuts of the MC_WWJETS truth analysis provided with the Rivet Monte-Carlo validation framework [66]. Specifically, we require charged leptons with $p_{\mathrm{T}, \ell}>25 \mathrm{GeV}$ and $\left|\eta_{\ell}\right|<3.5$. Missing transverse energy is identified with the vector sum of the neutrino transverse momenta and required to fulfil $E_{T}>25 \mathrm{GeV}$. Jets are defined using the anti- $k_{\mathrm{T}}$ algorithm [67] with a distance parameter of $R=0.4$. No jet-rapidity cuts are applied.

To illustrate the importance of the various corrections and the respective scale uncertainties, we present cross sections and distributions at the different levels of simulation introduced in section 3.3. In section 4.1 we compare fixed-order predictions to matched and merged NLO simulations. Squared quark-loop corrections are discussed in section 4.2.

\subsection{Fixed-order, matched and merged NLO simulations}

Rates for the inclusive analysis and when requiring (at least) one jet with $p_{\mathrm{T}}>30 \mathrm{GeV}$ are shown in table 2. Fixed-order LO and NLO predictions for pp $\rightarrow 4 \ell$ or $4 \ell+1 j$, depending on the jet bin, are compared to the inclusive MC@NLO $4 \ell$ simulation and to the NLO merged simulation of $4 \ell+0,1 j$. For 0 - and 1 -jet production we observe positive NLO corrections of $50 \%$ and $38 \%$, respectively, consistent with the typical size of $K$-factors in the literature. At NLO, scale uncertainties range from 3 to 5 percent, which is twice as

\footnotetext{
${ }^{5}$ Another natural way of combining these two sources of uncertainty is to consider simultaneous variations of $\left(\mu_{\mathrm{R}}, \mu_{\mathrm{F}}, \mu_{Q}\right)$, excluding rescalings in opposite directions as usual. The variations resulting from this alternative approach are likely to be even smaller than those obtained by adding QCD- and resummationscale uncertainties in quadrature.

${ }^{6}$ This pre-release version corresponds to SVN revision 21825 and the main difference with respect to the final SHERPA 2.0 release version is the tuning of parton shower, hadronisation and multiple parton interactions to experimental data.
} 


\begin{tabular}{|c|c|c|c|c|}
\hline & & & $4 \ell$ & MePs@NLO $4 \ell+0,1 j$ \\
\hline & $217.99(2){ }_{-2.8 \%}^{+1.9 \%}$ & & $70(29)_{-}^{+}$ & $356.01(58){ }_{-0.8 \%}^{+1.3 \%}{ }_{-0.0 \%}^{+1.8 \%}$ \\
\hline$\geq 1$ & $73.61(1){ }_{-11.6 \%}^{+14.5 \%}$ & $101.70_{-4.9 \%}^{+5.2 \%}$ & $83.23(15) \begin{array}{l}+9.9 \%+2.4 \% \\
-9.0 \%-4.6 \%\end{array}$ & $103.45(28)_{-3.7 \%}^{+2.8 \%}{ }_{-0.5 \%}^{+3.3 \%}$ \\
\hline
\end{tabular}

Table 2. Cross-section predictions in femtobarns for the $\mu^{+} \nu_{\mu} \mathrm{e}^{-} \bar{\nu}_{\mathrm{e}}$ analyses requiring $\geq 0$ and $\geq 1$ jets. Fixed-order LO and NLO results for the $\geq 0$-jet and $\geq 1$-jet analyses correspond to $4 \ell$ and $4 \ell+1 j$ production, respectively. They are compared to an inclusive Mc@NLO $4 \ell$ simulation and to MEPS@NLO 4 $+0,1 j$ predictions. Uncertainties associated to variations of the QCD scales $\left(\mu_{\mathrm{R}}, \mu_{\mathrm{F}}\right)$ and the resummation scale $\left(\mu_{Q}\right)$ are shown separately as $\sigma \pm \delta_{\mathrm{QCD}} \pm \delta_{\text {res }}$. Statistical errors are given in parenthesis.

large as compared to our previous Higgs-background predictions in exclusive jet bins [31]. This can be attributed to the new scale choice (3.5) and to the fact that results in table 2 correspond to inclusive jet bins. In fact, as shown in ref. [17], the choice of the central scale and a jet veto can have a strong impact on scale uncertainties in $4 \ell+1 j$ production [17]. In this respect, we note that the central scale used in ref. [31], i.e. the total four-lepton invariant mass, is more than a factor two higher than the transverse-energy scale (3.5) adopted for the present study.

Comparing the MC@NLO and NLO simulations we observe one-percent level agreement and rather similar uncertainties in the inclusive analysis. This agreement, as well as the tiny resummation-scale uncertainties of MC@NLO, reflect the unitarity of the parton shower for inclusive observables. In contrast, in the 1-jet bin MC@NLO predictions exhibit a deficit of about $20 \%$ and much larger uncertainties as compared to NLO. This is due to the fact that the inclusive matched calculation is only LO accurate in the 1-jet bin.

The inclusive MePs@NLO cross section is found to be roughly 30 fb larger as compared to the NLO calculation, which can be interpreted as a result of NLO corrections to the first emission in the merged sample. In fact, the shift of $30 \mathrm{fb}$ is comparable to the difference between the NLO and MC@NLO cross sections with $\geq 1$ jets, which corresponds to NLO effects in the 1-jet bin. Finally, variations of the QCD and resummation scales in MePs@Nlo amount to only 1-3\% in both jet bins. As already mentioned, the fact that fixed-order NLO cross sections feature significantly larger scale variations is related to the choice of the central scale $\mu_{0}$. This scale plays only a marginal role in MEPS@NLO, since the $\mathrm{pp} \rightarrow 4 \ell$ core process does not depend on the strong coupling, and $\alpha_{s}$ terms resulting from jet emissions are controlled by the CKKW prescription.

Distributions in the hardest-jet transverse momentum and in the total transverse energy $H_{\mathrm{T}}$ - defined as the scalar sum of the transverse momenta of leptons, missing $E_{\mathrm{T}}$, and all reconstructed jets - are displayed in figure 3. The bands are obtained by adding QCD- and resummation-scale variations in quadrature. The MC@NLO and MePs@NLO $p_{\mathrm{T}}$-distributions agree fairly well in the soft region, but MC@NLO develops an increasingly large deficit at higher $p_{\mathrm{T}}$, which reaches $30 \%$ in the tail. Similarly as Mc@NLO, also NLO predictions for inclusive four-lepton production are only LO accurate in the first-jet emission and tend to underestimate the tail. The shapes of the Mc@NLO and NLO tails 

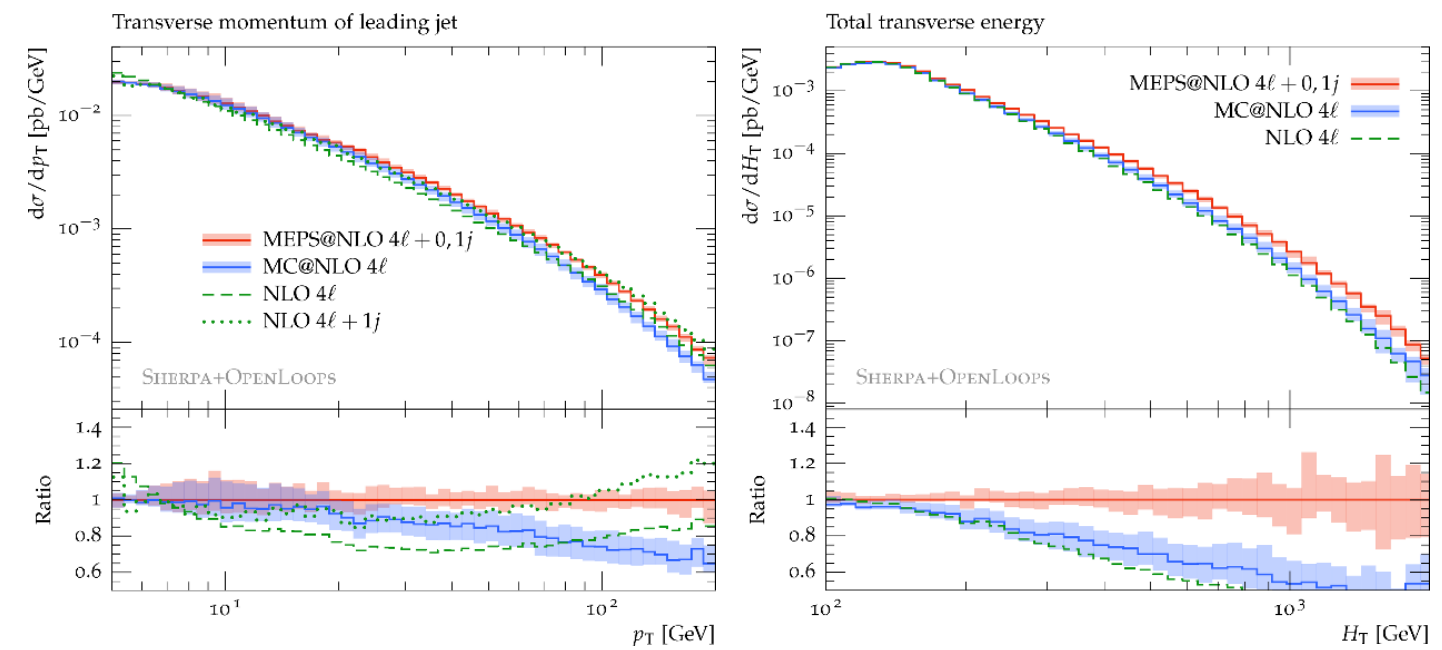

Figure 3. Leading-jet transverse momentum (left) and total transverse energy (right): NLO $4 \ell$ (green dashed) and NLO $4 \ell+1 j$ (green dotted) results are compared to an inclusive MC@NLO $4 \ell$ simulation (blue) and to MEPS@NLO $4 \ell+0,1 j$ predictions (red). Uncertainty bands describe combined QCD- and resummation-scale uncertainties (added in quadrature).

are however somewhat different. This is due to the fact that, in the MC@NLO method, the weights of the first shower emission and of its MC-subtraction counterpart differ by an $\mathcal{O}\left(\alpha_{s}\right)$ relative factor, which involves the $\alpha_{s}\left(p_{\mathrm{T}}\right) / \alpha_{s}\left(\mu_{\mathrm{R}}\right)$ ratio as well as unresolved NLO corrections. This difference disappears above the resummation scale, i.e. where the parton shower stops emitting. This is however not visible in the plot, since due to the dynamical nature of the resummation-scale choice (3.5), this transition takes place only far above the scale $M_{\mathrm{W}}$. In the $p_{\mathrm{T}} \rightarrow 0$ limit, the NLO $4 \ell$ calculation involves an infrared singularity of the form $\mathrm{d} \sigma / \mathrm{d} p_{\mathrm{T}} \sim \alpha_{s} \ln \left(p_{\mathrm{T}}\right) / p_{\mathrm{T}}$, which manifests itself as a linear rise if the distribution is plotted against $\ln \left(p_{\mathrm{T}}\right)$ as in figure 3.a. This feature is qualitatively clearly visible but quantitatively very mild, and the corresponding enhancement does not exceed $20 \%$ down to $p_{\mathrm{T}}=5 \mathrm{GeV}$. This signifies that the effect of resumming Sudakov logarithms is important but not dramatic in the considered $p_{\mathrm{T}}$-range. Higher Sudakov logarithms are partially included in the NLO calculation of $4 \ell+1 j$ production, which remains infrared divergent at $p_{\mathrm{T}} \rightarrow 0$, but turns out to be in better agreement with MC@NLO and MePs@NLO predictions for $p_{\mathrm{T}}>5 \mathrm{GeV}$. The NLO $4 \ell+1 j$ distribution has a higher tail with respect to inclusive NLO and MC@NLO predictions, as expected, but for $p_{\mathrm{T}} \gtrsim M_{\mathrm{W}}$ it starts to be above the MePs@NLO curve as well. This can be explained by the fact that, in contrast to the MePs@NLO approach, in fixed-order predictions the scale of $\alpha_{s}$ couplings associated with jet emission is not adapted to the jet- $p_{\mathrm{T}}$ (cf. discussion in section 2.3).

The total transverse energy, plotted in figure 3.b, is dominated by hard multi-jet emissions that cannot be properly described neither by the inclusive NLO calculation nor by the MC@NLO approach and its parton-shower emissions. This starts to be visible at $H_{\mathrm{T}} \sim 200 \mathrm{GeV}$ and the deficit with respect to MePs@NLO approaches $50 \%$ at $1 \mathrm{TeV}$.

Matching and merging effects in presence of a jet veto and jet binning are illustrated in figure 4 , where the integrated cross sections in the exclusive 0 -jet bin $\left(p_{\mathrm{T}}<p_{\mathrm{T}}^{\max }\right)$ and 
in the inclusive 1-jet bin $\left(p_{\mathrm{T}}>p_{\mathrm{T}}^{\mathrm{min}}\right)$ are plotted as a function of the corresponding upper and lower transverse-momentum bounds. In the 0-jet bin, MC@NLO and MEPs@NLO predictions agree well at small jet-veto scales and differ by less than $10 \%$ at large $p_{\mathrm{T}}^{\max }$. The respective uncertainties are as small as a few percent and nearly independent of $p_{T}^{\max }$. For sufficiently inclusive jet-veto values, the NLO pp $\rightarrow 4 \ell$ calculation is in excellent agreement with MC@NLO. In the $p_{\mathrm{T}}^{\max } \rightarrow 0$ limit, NLO predictions develop a double-logarithmic singularity of the form $-\alpha_{s} \ln ^{2}\left(p_{\mathrm{T}}^{\max } / Q\right)$, while Mc@NLO and MePs@NLO vetoed cross sections consistently tend to zero as a result of the exponentiation of Sudakov logarithms. In this infrared regime, the exponentiation of double logarithms should manifest itself as a positive correction beyond NLO, while for $p_{\mathrm{T}}^{\max } \gtrsim 10 \mathrm{GeV}$ we observe that matched/merged predictions are still below the NLO jet-vetoed cross section. This is due to the fact that Sudakov logarithms are relatively mild in this region (cf. figure 3.a), and parton-shower effects are dominated by subleading logarithms associated with the running of $\alpha_{s}$ in the $\alpha_{s}\left(p_{\mathrm{T}}\right) \ln \left(p_{\mathrm{T}}\right) / p_{\mathrm{T}}$ terms. Double logarithms become dominant at much smaller transverse momenta, and we checked that they drive the NLO cross sections into the negative range only at $p_{\mathrm{T}}^{\max } \sim 2 \mathrm{GeV}$. For $p_{\mathrm{T}}^{\max } \simeq 25-30 \mathrm{GeV}$, which corresponds to the jet-veto values in the $\mathrm{H} \rightarrow \mathrm{WW}^{*}$ analyses at the LHC, fixed-order and matched/merged results deviate by less than $5 \%$. This represents the net effect of Sudakov logarithms beyond NLO, and its smallness is due to the moderate size of the logarithmic terms but also to cancellations between leading and subleading logarithms. The uncertainty due to subleading Sudakov logarithms that are not included in the MC@NLO and MEPS@NLO approximations are quantified via resummation-scale variations, which are reflected in the respective scalevariation bands, and turn out to be at the percent level.

As shown in figure 4.b, in the inclusive 1-jet bin the discrepancies between the various approximations become more sizable. The inclusive MC@NLO simulation underestimates the 1-jet cross section by $20-30 \%$ for $30 \mathrm{GeV}<p_{\mathrm{T}}^{\min }<100 \mathrm{GeV}$. For transverse-momentum thresholds up to $50 \mathrm{GeV}$, the fixed-order $4 \ell+1 j$ cross section is in quite good agreement with the MePs@NLO prediction as expected. However, as already observed in figure 3.a, the NLO cross section develops a significant excess in the tail. The uncertainties of the MEPS@NLO and MC@NLO cross sections in the 1-jet bin are rather independent of the $p_{\mathrm{T}}$-threshold and amount to about $5 \%$ and $10 \%$, respectively.

\subsection{Squared quark-loop contributions}

Detailed results for the squared quark-loop cross sections in the inclusive analysis and requiring one or more jets with $p_{\mathrm{T}}>30 \mathrm{GeV}$ are presented in table 3. Fixed-order calculations for $4 \ell$ or $4 \ell+1 j$ production, depending on the jet bin, are compared to an inclusive simulation obtained by showering four-lepton matrix elements (LoOP ${ }^{2}+\mathrm{PS} 4 \ell$ ) and to merged predictions (MePs@Loop $24 \ell+0,1 j$ ). Additionally, to assess the importance of quark-induced channels, we show merged squared quark-loop results that involve only gluon-gluon partonic channels and, for consistency, only $g \rightarrow$ gg splittings in the parton shower.

As compared to the MEPS@NLO cross sections in table 2, squared quark loops represent a correction of about $3 \%$, both in the inclusive analysis and in the 1-jet bin. In 

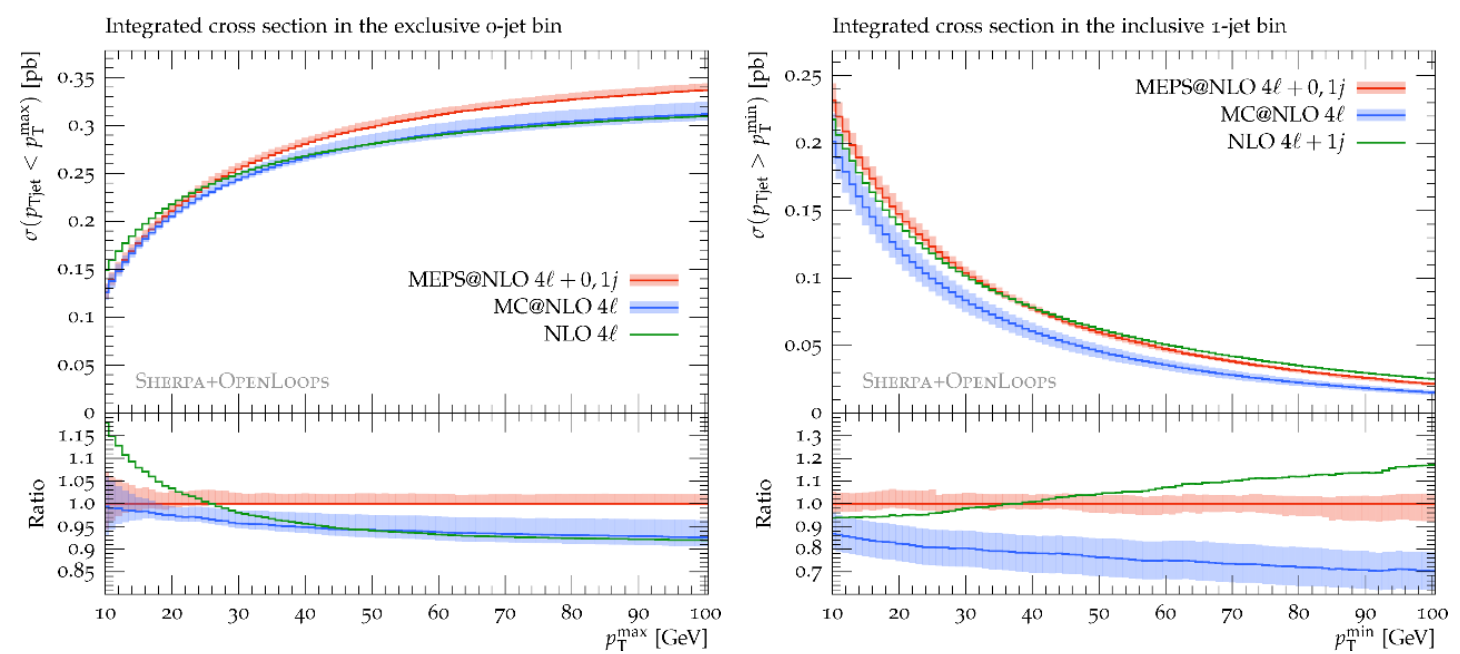

Figure 4. Integrated cross sections in the exclusive 0-jet bin (left) and in the inclusive 1-jet bin (right) as a function of the respective transverse-momentum bounds, $p_{\mathrm{T}}^{\max }$ and $p_{\mathrm{T}}^{\min }$. NLO results with appropriate jet multiplicity (green) are compared to MC@NLO $4 \ell$ (blue) and MEPS@NLO $4 \ell+$ $0,1 j$ (red) simulations. Uncertainty bands correspond to QCD-scale variations combined with the resummation-scale variations in quadrature.

\begin{tabular}{|c|c|c|c|c|}
\hline Analysis & $\operatorname{LoOP}^{2} 4 \ell(+1 j)$ & $\mathrm{LOOP}^{2}+\mathrm{PS} 4 \ell$ & $\begin{array}{l}\text { MEPS@LOOP } \\
\mathrm{pp} \rightarrow 4 \ell+0,1 j\end{array}$ & $\begin{array}{l}\text { MEPS@LOOP } \\
\mathrm{gg} \rightarrow 4 \ell+0,1 \mathrm{~g}\end{array}$ \\
\hline$\geq 0$ jets & $8.71(3)+28 \%$ & $\begin{array}{l}8.76(3)+28 \%+0.2 \% \\
-21 \%-0.1 \%\end{array}$ & $\begin{array}{l}9.24(4)+31 \%+20 \% \\
-20 \%-14 \%\end{array}$ & $\begin{array}{c}9.10(3)+28 \%+15 \% \\
-21 \%-12 \%\end{array}$ \\
\hline$\geq 1$ jets & $3.98(7)_{-30 \%}^{+48 \%}$ & $1.75(1) \begin{array}{l}+32 \%+55 \% \\
-25 \%\end{array}$ & $2.75(3){ }_{-24 \%}^{+40 \%}+5 \%$ & $2.01(2)+35 \%+1.4 \%$ \\
\hline
\end{tabular}

Table 3. Squared quark-loop predictions in femtobarns for the $\mu^{+} \nu_{\mu} \mathrm{e}^{-} \bar{\nu}_{\mathrm{e}}$ analyses requiring $\geq 0$ jets and $\geq 1$ jets. Fixed-order results $\left(\mathrm{LOOP}^{2}\right)$ with a number of jets corresponding to the actual analysis are compared to an inclusive parton-shower simulation ( $\operatorname{LoOP}^{2}+\mathrm{PS} 4 \ell$ ) and to predictions from the merged MEPs@Loop ${ }^{2} 4 \ell+0,1 j$ simulation with and without the inclusion of quarks in the initial state. Scale variations and statistical errors are presented as in table 2.

the inclusive case, fixed-order and shower-improved predictions are in excellent agreement, as expected from the unitarity of the shower. In contrast, the $\mathrm{LOOP}^{2}+\mathrm{PS}$ simulation - which corresponds to the approach typically adopted in present experimental studies, where jet emission is entirely based on the shower approximation — underestimates the squared quark-loop cross section in the inclusive 1-jet bin by around $50 \%$. Due to their LO $\alpha_{s}^{2}$ and $\alpha_{s}^{3}$ dependence, squared quark-loop corrections feature a QCD-scale dependence of $30-40 \%$. The resummation-scale uncertainty of the $\mathrm{LOOP}^{2}+\mathrm{PS}$ simulation is close to zero in the inclusive case (due to unitarity), while in the 1-jet bin it is as large as $50 \%$, due to the fact that the 1-jet bin is entirely filled by shower emissions.

Comparing LoOP ${ }^{2}+\mathrm{PS}$ predictions to the merged sample we observe that the matrixelement description of jet emission significantly increases the cross section, especially in the 1-jet bin. The QCD-scale uncertainty remains at 30-40\% level, but resummation-scale variations change substantially: the 1-jet bin cross section becomes almost independent of 
the resummation scale, since, as a result of merging, 1-jet events are described in terms of matrix elements, and shower emissions induce only minor bin migrations. In contrast, in the inclusive analysis the merged simulation features a significantly higher resummationscale dependence of approximately $15 \%$, which can be attributed to unitarity violations induced by the merging procedure: the resummation-scale dependence that arises from the region below the merging cut, where 0-jet matrix elements are combined with the Sudakov suppression factor, is not compensated by an opposite dependence from above $Q_{\text {cut }}$, since the parton shower is superseded by 1-jet matrix elements in that region. We note that this kind of resummation-scale sensitivity is due to the LO nature of squared quark-loop merging and is strongly reduced in the case of NLO merging (cf. last column in table 2). The fact that the MePs@Loop ${ }^{2}$ cross section in the 1-jet bin is $30 \%$ below the fixedorder result can be attributed to the CKKW scale choice in the merging approach and is consistent with the size of renormalisation-scale variations. Finally, comparing the last two columns in table 3 , we observe that quark-induced channels account for roughly $1.5 \%$ and $30 \%$ of the squared quark-loop corrections in the 0- and 1-jet bins, respectively. This corresponds to about 0.5 permil and 1 percent of the total cross section in the respective jet bins. We note that the individual impact of quark channels at matrix-element or partonshower level is significantly larger, i.e. a naive merging approach based on pure-gluon matrix elements plus a standard parton shower would lead to bigger deviations with respect to the Meps@Loop ${ }^{2}$ results in table 3.

Squared quark-loop corrections to differential observables are compared to NLO merged predictions in figure 5. As already found in tables 2 and 3, their impact typically amounts to a few percent. Both for the leading-jet transverse momentum and for the dilepton invariant mass they feature a rather different kinematic dependence as compared to MEPS@NLO results. In the considered range their relative importance varies from one to seven percent, and the maximum lies in the region of small dilepton mass, which corresponds to the signal region of the $\mathrm{H} \rightarrow \mathrm{WW}^{*}$ analysis.

Merging effects are illustrated in the left plot of figure 6, where predictions from the inclusive squared quark-loop gg $\rightarrow 4 \ell$ matrix element supplemented with a regular parton shower $\left(\mathrm{LOOP}^{2}+\mathrm{PS}\right)$ are compared to the merged pp $\rightarrow 4 \ell+0,1 j$ simulation $\left(\right.$ MePs@Loop ${ }^{2}$ ). The latter is decomposed into contributions from $4 \ell+0 j$ and $4 \ell+1 j$ matrix elements. In the region well below the merging cut, $Q_{\text {cut }}=20 \mathrm{GeV}$, merged predictions are dominated by 0 -jet matrix elements and agree almost perfectly with the LoOP ${ }^{2}+\mathrm{PS}$ curve. The agreement remains better than $10 \%$ up to $p_{\mathrm{T}} \sim Q_{\text {cut }}$, where the MePS@Loop ${ }^{2}$ sample is characterised by the transition from the 0 -jet to the 1 -jet matrix-element regime. This supports the use of the 0 -jet plus shower approximation up to the merging scale. Starting from $p_{\mathrm{T}} \gtrsim 40 \mathrm{GeV}$, where 1-jet matrix elements dominate and render MEPS@Loop ${ }^{2}$ predictions more reliable, the parton-shower results feature a sizable deficit and are also strongly sensitive to the resummation scale.

Setting the resummation scale equal to the default scale (3.5), we found that the slight excess of the parton shower at $p_{\mathrm{T}} \sim Q_{\text {cut }}$ propagates to higher transverse momenta reaching up to $40 \%$ at $p_{\mathrm{T}} \gtrsim 100 \mathrm{GeV}$. In order to avoid such an unnatural parton-shower excess at high $p_{\mathrm{T}}$, and a corresponding excess in the Sudakov suppression at low $p_{\mathrm{T}}$, as 

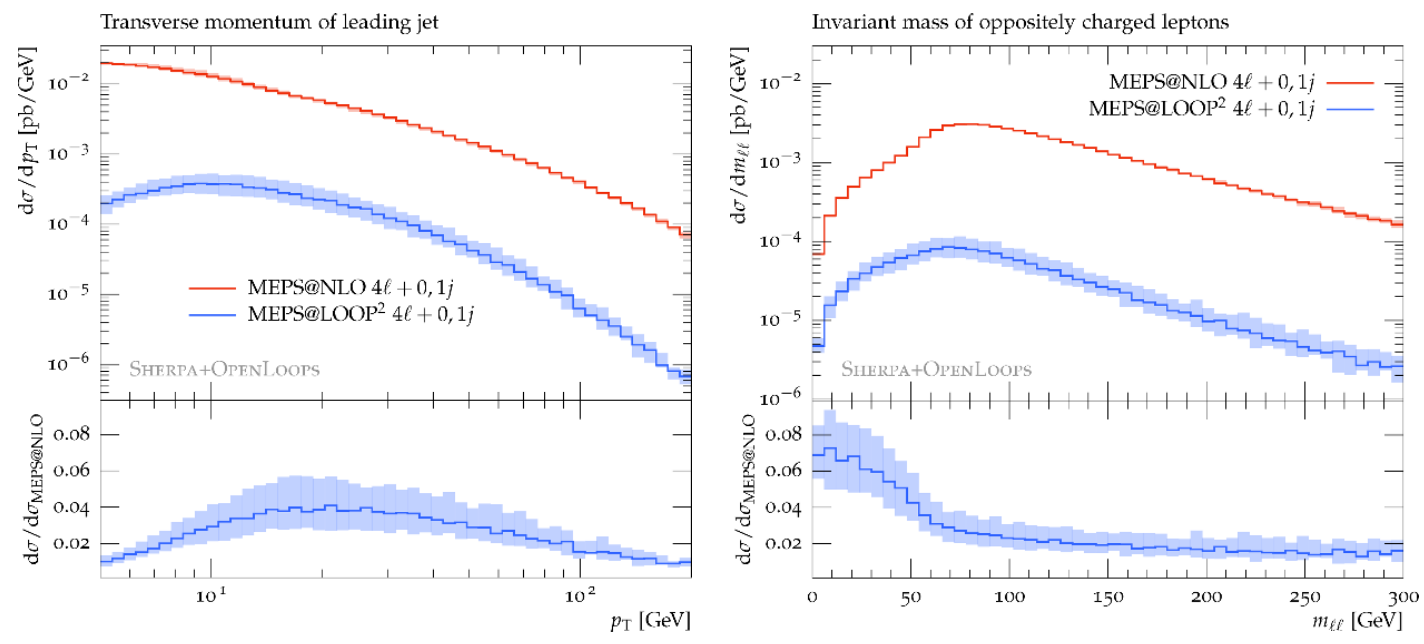

Figure 5. Comparison of merged squared quark-loop (blue) and NLO (red) predictions for $4 \ell+0,1 j$ production: transverse momentum of the leading jet (left) and invariant mass of the two charged leptons (right).
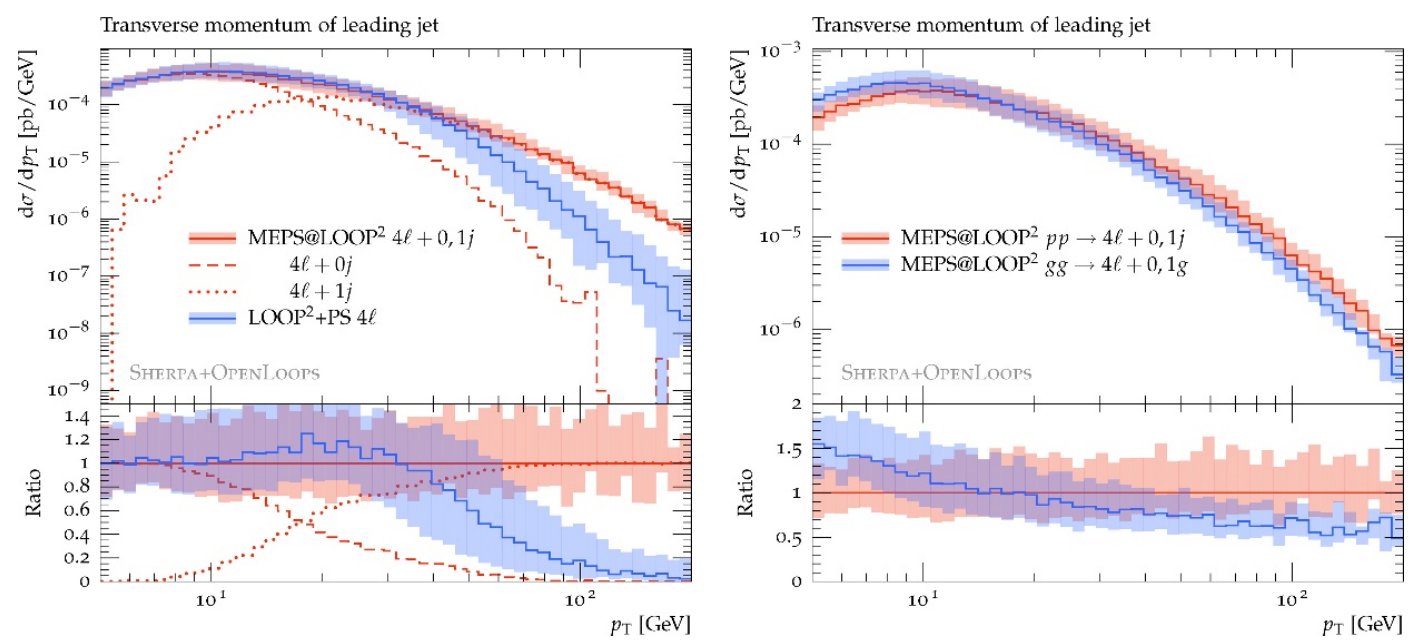

Figure 6. Squared quark-loop corrections to the leading-jet $p_{\mathrm{T}}$-distribution: (a) a simulation based on $4 \ell$ matrix elements plus parton shower (blue) is compared to complete merged predictions (red solid). The latter are split into the contributions from $4 \ell+0 j$ (red dashed) and $4 \ell+1 j$ (red dotted) matrix elements; (b) full merged predictions (red) are compared to a corresponding simulation involving only gluon contributions (blue). Uncertainty bands correspond to the combination (in quadrature) of QCD- and resummation-scale variations.

anticipated in section 4.1 we decided to evaluate squared quark-loop contributions using a smaller resummation scale, $\mu_{Q}=\mu_{0} / 2$. Of course the small value of $\mu_{Q}$ amplifies the natural deficit of the shower at large $p_{\mathrm{T}}$ and yields a quite small $\mathrm{LOOP}^{2}+\mathrm{PS}$ cross section in the 1-jet bin (cf. table 3). However this side-effect is compensated by 1-jet matrix elements in the MePs@Loop ${ }^{2}$ simulation. The bands describe the total scale uncertainty, obtained by adding QCD- and resummation-scale variations in quadrature. Apart from the suppressed high- $p_{\mathrm{T}}$ tail of the $\mathrm{LOOP}^{2}+\mathrm{PS}$ distribution, we find a rather constant uncertainty of about $30 \%$. 

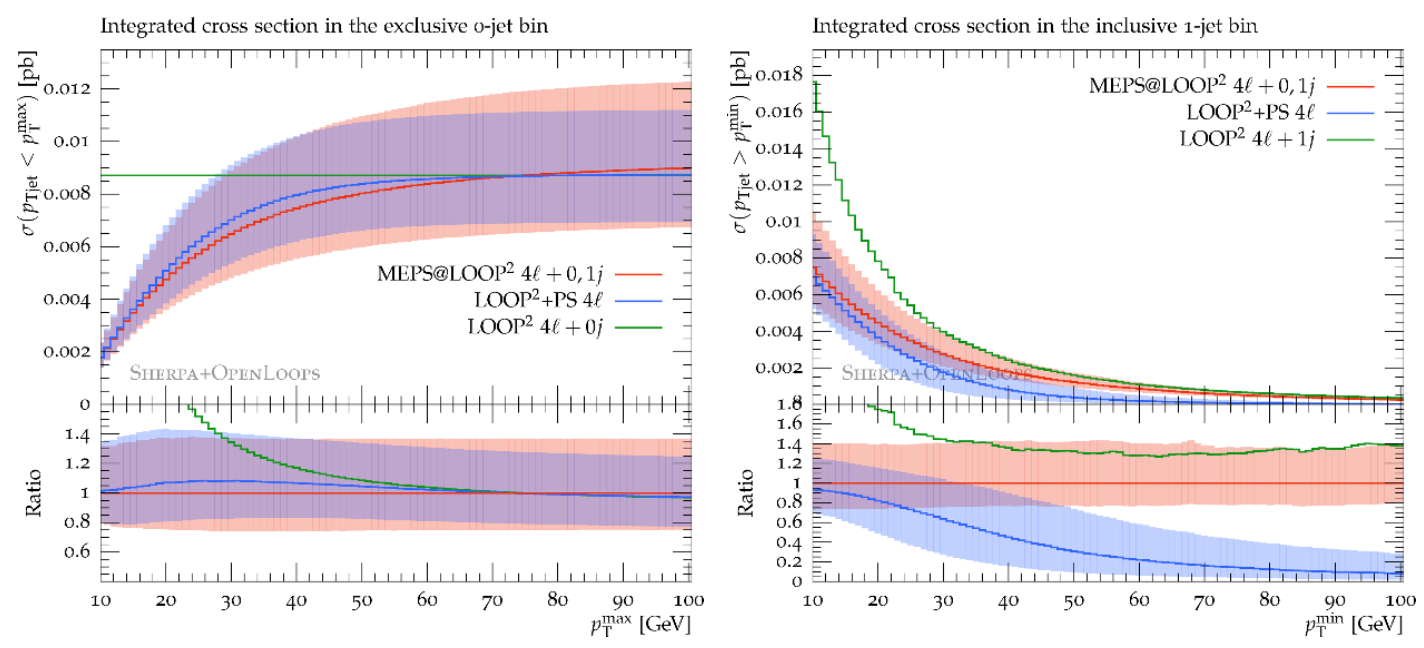

Figure 7. Integrated squared quark-loop cross sections in the exclusive 0-jet bin (left) and in the inclusive 1-jet bin (right) as a function of the respective transverse-momentum bounds, $p_{\mathrm{T}}^{\max }$ and $p_{\mathrm{T}}^{\mathrm{min}}$. Fixed-order $\mathrm{LoOP}^{2} 4 \ell(+1 j)$ results (green) are compared to LooP ${ }^{2}+\mathrm{PS} 4 \ell$ (blue) and MEPS@LOOP $24+0,1 j$ (red) simulations. Uncertainty bands correspond to QCD-scale variations combined with resummation-scale variations in quadrature.

The right plot in figure 6 illustrates the impact of quark-channel contributions on the leading-jet $p_{\mathrm{T}}$-distribution. Plotted are full MePs@Loop ${ }^{2}$ results and corresponding predictions involving only gg-induced matrix elements and $g \rightarrow$ gg shower splittings. As is clearly visible from the ratio plot, the quark channels enhance hard-jet emissions and induce a related Sudakov suppression at low $p_{\mathrm{T}}$. The resulting distortion in the jet- $p_{\mathrm{T}}$ distribution amounts to $\pm 50 \%$. When looking at table 3 , such opposite behaviour in the hard and soft regions explains why the quark-channel contribution reaches $30 \%$ in the 1-jet bin but goes down to $1.5 \%$ in the inclusive case.

Jet-veto and jet-binning effects on squared quark-loop contributions are shown in figure 7 , where the integrated cross sections in the exclusive 0 -jet bin $\left(p_{\mathrm{T}}<p_{\mathrm{T}}^{\max }\right)$ and in the inclusive 1-jet bin $\left(p_{\mathrm{T}}>p_{\mathrm{T}}^{\min }\right)$ are plotted as a function of $p_{\mathrm{T}}^{\max }$ and $p_{\mathrm{T}}^{\min }$. In the 0 -jet bin, apart from the minor excess around $30 \mathrm{GeV}$, LoOP ${ }^{2}+\mathrm{PS}$ predictions agree quite well with Meps@Loop ${ }^{2}$ ones for any jet-veto scale up to $100 \mathrm{GeV}$. The corresponding scale uncertainties are in the $20-40 \%$ range. As in table 3, Meps@Loop ${ }^{2}$ uncertainties tend to be larger in the inclusive limit. Fixed-order gg $\rightarrow 4 \ell$ contributions are inherently inclusive and independent of $p_{\mathrm{T}}^{\max }$. Comparing them to the Meps@Loop ${ }^{2}$ and $\mathrm{LoOP}^{2}+\mathrm{PS}$ curves we observe that jet-veto scales of $25-30 \mathrm{GeV}$, as those used in the experimental $\mathrm{H} \rightarrow \mathrm{WW}^{*}$ analyses, correspond to a moderate cross-section suppression of approximately $30 \%$. In this regime the parton shower should provide a sufficiently reliable resummation of Sudakov logarithms.

The right plot of figure 7 compares fixed-order, shower-improved and merged predictions in the inclusive 1-jet bin. For a jet threshold of $30 \mathrm{GeV}$, the various approximations agree only marginally within the respective errors, while higher and smaller values of $p_{\mathrm{T}}^{\min }$ lead to very large discrepancies. As compared to MEPS@Loop ${ }^{2}$ predictions, at large $p_{\mathrm{T}}$ 
we observe a dramatic deficit of the shower approximation, while the fixed-order squared quark-loop calculation yields a rather constant $40 \%$ excess as in table 3 . The resummation of Sudakov logarithms becomes relevant only for transverse-momentum thresholds below $30 \mathrm{GeV}$, where the excess of the fixed-order prediction grows up to $150 \%$ at $10 \mathrm{GeV}$.

\section{Atlas and CMS $\mathrm{H} \rightarrow \mathrm{WW}^{*}$ analyses in the 0- and 1-jet bins}

In this section we study the irreducible four-lepton background to the ATLAS [3] and CMS [4] $H \rightarrow \mathrm{WW}^{*} \rightarrow \mu^{+} \nu_{\mu} \mathrm{e}^{-} \bar{\nu}_{\mathrm{e}}$ analyses at $8 \mathrm{TeV}$. We restrict ourselves to the exclusive 0- and 1-jet bins, which contain the bulk of the four-lepton background associated with diboson production, and focus on opposite-flavour $\mu^{+} \nu_{\mu} \mathrm{e}^{-} \bar{\nu}_{\mathrm{e}}+$ jets final states, which provide the highest sensitivity to the Higgs-boson signal. Technically, within the automated SHERPA+OPEnLoops framework, the simulation of $\ell \nu \ell \nu+$ jets production with same lepton flavour is almost equivalent to the opposite-flavour case. Also for what concerns QCD corrections and uncertainties we do not expect any important difference between oppositeand same-flavour channels.

In the following we apply the cuts listed in appendix B, which correspond to the AtLAS [3] and CMS [4] analyses at $8 \mathrm{TeV}$. Let us remind that the two experiments employ different definitions of the WW transverse mass, reported in eq. (B.1), and different anti$k_{\mathrm{T}}$ jet radii. Note also that ATLAS employs a lower transverse-momentum threshold for central jets. After a pre-selection, which basically requires two hard leptons and large missing energy, two complementary selections based on $p_{\mathrm{T}, \ell \ell^{\prime}}, \Delta \phi_{\ell \ell^{\prime}}, m_{\ell \ell^{\prime}}$ and $m_{T}$, are used to define a signal and a control region. The latter is exploited to normalise WWbackground simulations to data. Separate analyses are performed in the 0-, 1-, and 2-jet bins in order to improve the sensitivity to the Higgs-boson signal and the data-driven normalisation of the various background components.

In section 5.1 we investigate kinematic distributions that are relevant for the experimental selection after pre-selection cuts. In section 5.2 we consider the control and signal regions and discuss the observables that are exploited in the final stage of the Higgs analyses, namely the WW transverse mass and the dilepton invariant mass. Finally, in section 5.3 we present predictions for the 0 - and 1 -jet bin cross sections in the signal and control regions, as well as uncertainties associated with variations of renormalisation, factorisation, resummation, and merging scales.

For each observable we present results for the ATLAS and CMS analyses in the exclusive 0 - and 1-jet bins and, to provide insights into the convergence of the perturbative expansion and the size of Sudakov logarithms in jet bins, we compare NLO, MC@NLO, MEPs@NLO and squared quark-loop predictions. As discussed in section 3, in NLO predictions for the 0and 1-jet bins we always include the corresponding number of jets at matrix-element level. In contrast, MC@NLO results refer as usual to a single simulation of inclusive $\mu^{+} \nu_{\mu} \mathrm{e}^{-} \bar{\nu}_{\mathrm{e}}$ production, which is NLO accurate in the 0-jet bin and only LO accurate in the 1-jet bin. Only MePs@NLO predictions are consistently matched to the parton shower and NLO accurate in both jet bins. 


\subsection{Kinematic distributions after pre-selection cuts}

In figures 8-10 we present jet and lepton observables after pre-selection cuts. The curves for Meps@Nlo, Mc@Nlo, Nlo, and Meps@Loop ${ }^{2}$ correspond to the central scale choice (3.5). The middle and lower panels show relative MC@NLO and NLO deviations from MEPS@NLO, and squared quark-loop contributions normalised to the central Meps@Nlo result. Scale-variation bands are shown only for MePs@Loop ${ }^{2}$ and MePs@NLO. In the latter case, renormalisation- and factorisation-scale variations $\Delta_{\mathrm{QCD}}$ (red band), resummation-scale variations $\Delta_{\text {res }}$ (blue band), and their combination in quadrature $\Delta_{\text {tot }}=\left(\Delta_{\text {QCD }}^{2}+\Delta_{\text {res }}^{2}\right)^{1 / 2}$ (yellow band), are displayed as colour-additive regions. The various band regions assume different colours corresponding to the various possible overlaps. The band boundary, corresponding to variations $\delta$ in the range $\Delta_{\mathrm{QCD}}, \Delta_{\text {res }}<\delta<\Delta_{\text {tot }}$, is yellow. Orange areas appear in kinematic regions dominated by QCD-scale variations $\left(\Delta_{\text {res }}<\delta<\Delta_{\mathrm{QCD}}\right)$, while green areas reflect dominant resummationscale variations $\left(\Delta_{\mathrm{QCD}}<\delta<\Delta_{\text {res }}\right)$, and the central band area $\left(\delta<\Delta_{\text {res }}, \Delta_{\mathrm{QCD}}\right)$, where all three colours overlap, is brown. Note that scale-variation bands are somewhat distorted by statistical fluctuations, which tend to increase in the tails of some distributions.

Before splitting the event sample into exclusive jet bins, in figure 8 we show the transverse momenta of the hardest (upper plots) and second-hardest (lower plots) jet. Here all NlO curves correspond to $4 \ell+1$ jet production. In the case of the first jet, MC@NLO predictions are only LO accurate and significantly underestimate the tail of the $p_{\mathrm{T}}$ distribution. On the other hand, NLO predictions feature a $20 \%$ excess at high $p_{\mathrm{T}}$. As already observed in figure 3.a, this behaviour can be explained by the fact that the scale (3.5) used in the fixed-order calculation does not adapt to the transverse momentum of the jet.

In the case of the second-jet $p_{\mathrm{T}}$, NLO and MEPS@NLO results are both only LO accurate, and the shape differences at large $p_{\mathrm{T}}$ are more pronounced but qualitatively similar as for the first jet. The excess of the NLO distribution below $10 \mathrm{GeV}$ reveals the presence of the infrared singularity at $p_{\mathrm{T}} \rightarrow 0$. The MC@NLO prediction for the second jet is entirely based on the shower approximation. It remains low over the entire spectrum, and above $30 \mathrm{GeV}$ the deficit starts to be considerable.

The increase of MEPS@NLO scale variations from a few percent for the first jet to 10\% for the second one, is due to the transition from NLO to LO accuracy. The abundance of orange and brown areas in the MEPS@NLO bands indicates that the uncertainty tends to be dominated by QCD-scale variations. Green band areas, which correspond to larger resummation-scale uncertainties, show up less frequently and only in the leading-jet $p_{\mathrm{T}}$ distribution. Even in the small- $p_{\mathrm{T}}$ region, where Sudakov logarithms have the highest possible impact, QCD- and resummation-scale variations do not exceed 10\%. This suggests that subleading-logarithmic corrections beyond the MEPS@NLO accuracy should be rather modest.

Squared quark-loop corrections range from 1 to 6 percent and feature a more pronounced dependence on the jet $p_{\mathrm{T}}$ as compared to the inclusive analysis (cf. figure 5). The largest effects arise around $p_{\mathrm{T}} \simeq 20 \mathrm{GeV}$, which corresponds to the 0 -jet bin of the $\mathrm{H} \rightarrow \mathrm{WW}^{*}$ analysis. 
Let us now switch to leptonic observables in the exclusive 0- and 1-jet bins of the $\mathrm{H} \rightarrow \mathrm{WW}^{*}$ analyses. Distributions in the azimuthal dilepton separation $\Delta \phi_{\ell \ell^{\prime}}$ and in the dilepton invariant mass $m_{\ell \ell^{\prime}}$ are displayed in figures 9 and 10 . These observables play an important role for the description of the background acceptance and for the optimisation of the Higgs-boson sensitivity in the experimental analyses. The corresponding MEPS@NLO distributions are NLO accurate in both jet bins. This is very well reflected by the MePs@NLO uncertainty bands, which do not exceed the few-percent level. Also here, resummation-scale variations tend to be slightly subdominant with respect to QCD-scale variations. Comparing Nlo, MC@NLO and MePs@Nlo distributions in the 0-jet bin, where none of these approximations loses NLO accuracy, we find overall agreement at the few-percent level. In the 1-jet bin, the agreement between NLO and MEPS@NLO remains, as expected, quite good. Due to the lack of NLO accuracy, inclusive MC@NLO predictions feature the characteristic 10-15\% deficit in the 1-jet bin, which is accompanied by minor shape distortions. Given the good agreement with NLO within the small uncertainty band, the shape of MEPS@NLO distributions seems to be very well under control.

In the 0 -jet bin, MEPs@Loop ${ }^{2}$ corrections are very sensitive both to the azimuthal separation and to the invariant mass of the dilepton system. At small $\Delta \phi_{\ell \ell^{\prime}}$ and $m_{\ell \ell^{\prime}}$, which corresponds to the Higgs-signal region, they reach up to $8 \%$ and $6 \%$, respectively. A similar but weaker sensitivity is visible also in the 1-jet bin.

Inspecting the transverse-momentum distributions of the harder and softer charged lepton (not shown here) we found that the various NLO corrections behave very similarly as for $\Delta \phi_{\ell \ell^{\prime}}$ and $m_{\ell \ell^{\prime}}$, while squared quark-loop corrections are less sensitive to the lepton$p_{\mathrm{T}}$ and vary between $2 \%$ and $4 \%$ only.

\subsection{Kinematic distributions in control and signal regions}

We now turn to the control (C) and signal (S) regions of the experimental analyses (see table 7) and discuss the distributions in the WW transverse mass, $m_{\mathrm{T}}$, and in the dilepton invariant mass, $m_{\ell \ell^{\prime}}$. These observables are sensitive to the Higgs-boson signal, and their shape permits to increase the signal-to-background discrimination in the final fit. Separate distributions for the exclusive 0- and 1-jet bins and for the two experiments are shown in figures 11-13.

In the signal and control regions, as well as in both jet bins, the size of the various corrections and the MEPs@NLO uncertainties behave fairly similar to what observed at pre-selection level. The NLO, MC@NLO and MePs@NLO distributions agree at fewpercent level in the 0-jet bin, while in the 1-jet bin discrepancies between MC@NLO and MePs@NLO on the 10-15\% level and little MC@NLO shape distortions appear. The size of the corrections and the scale uncertainties for the two experimental analyses are qualitatively and quantitatively similar. Obviously, due to the different cuts, absolute background predictions for AtLas and CMS behave differently. The shapes of MePs@Nlo distributions are again in excellent agreement with NLO, suggesting moderate Sudakov logarithms beyond NLO. This is consistent with the small scale uncertainty of the merged simulation.

Squared quark-loop corrections feature a nontrivial sensitivity to $m_{\mathrm{T}}$ and $m_{\ell \ell^{\prime}}$, which varies depending on the experimental analysis, the selection region, and the jet bin. The 

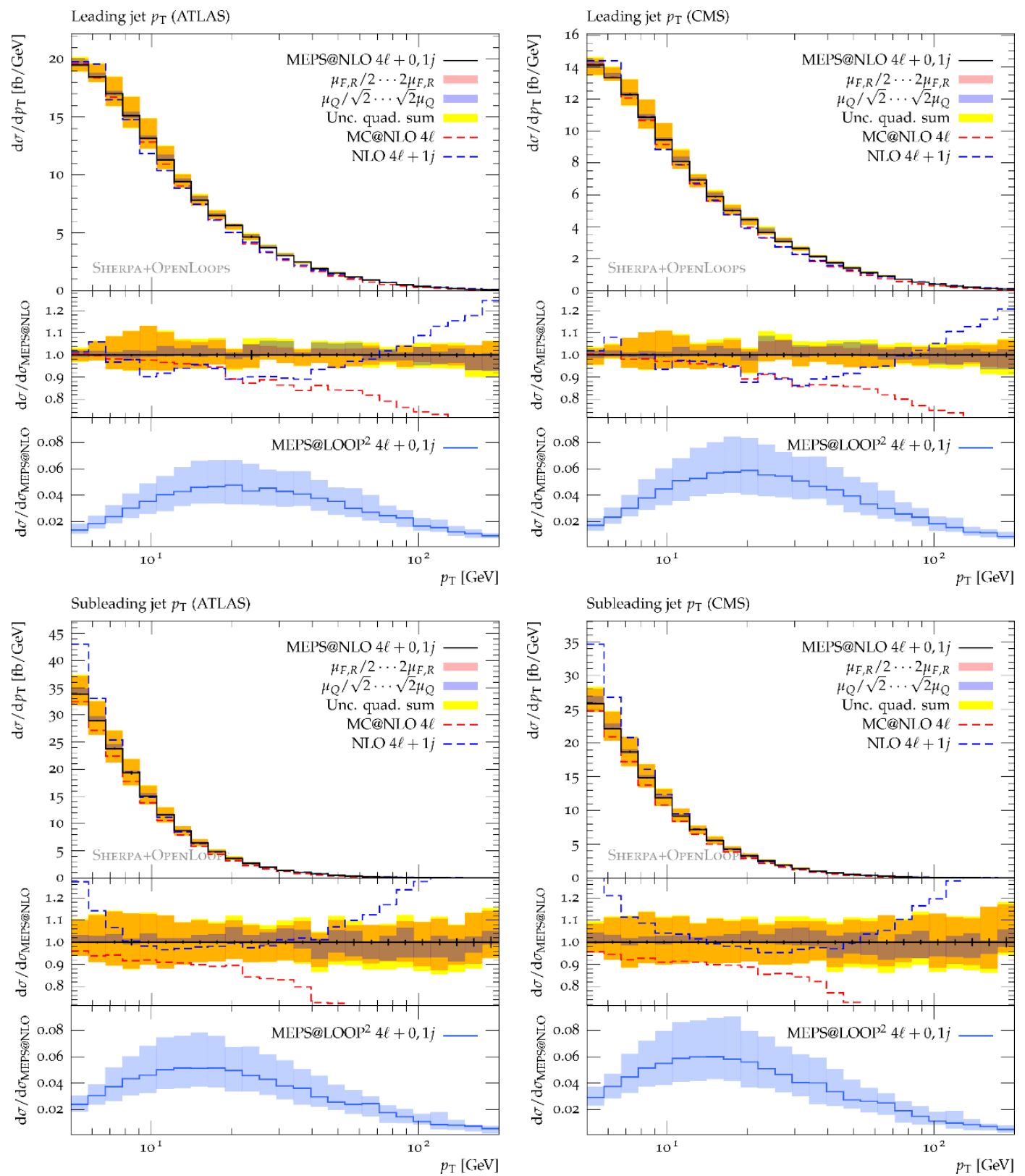

Figure 8. AtLAS (left) and CMS (right) analysis at $8 \mathrm{TeV}$ after pre-selection cuts: transversemomentum distributions of the first (top) and second (bottom) jet. MePs@Nlo (black solid), inclusive MC@NLO (red dashed), and NLO (blue dashed) predictions at the central scale. The ratio plots in the middle panels show relative uncertainties as well as MC@NLO and NLO deviations

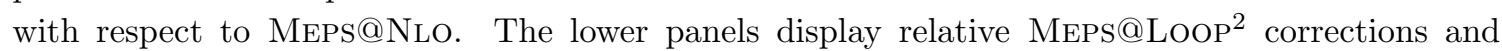
uncertainties normalised to MEPs@NLO at the central scale. The factor-two variations of $\mu_{\mathrm{R}}$ and $\mu_{\mathrm{F}}$ (red band), and factor $-\sqrt{2}$ variations of $\mu_{Q}$ (blue band), are combined in quadrature (yellow band). Scale-variation bands are colour additive, i.e. yellow + blue $=$ green, yellow + red $=$ orange, and yellow + red + blue $=$ brown. 

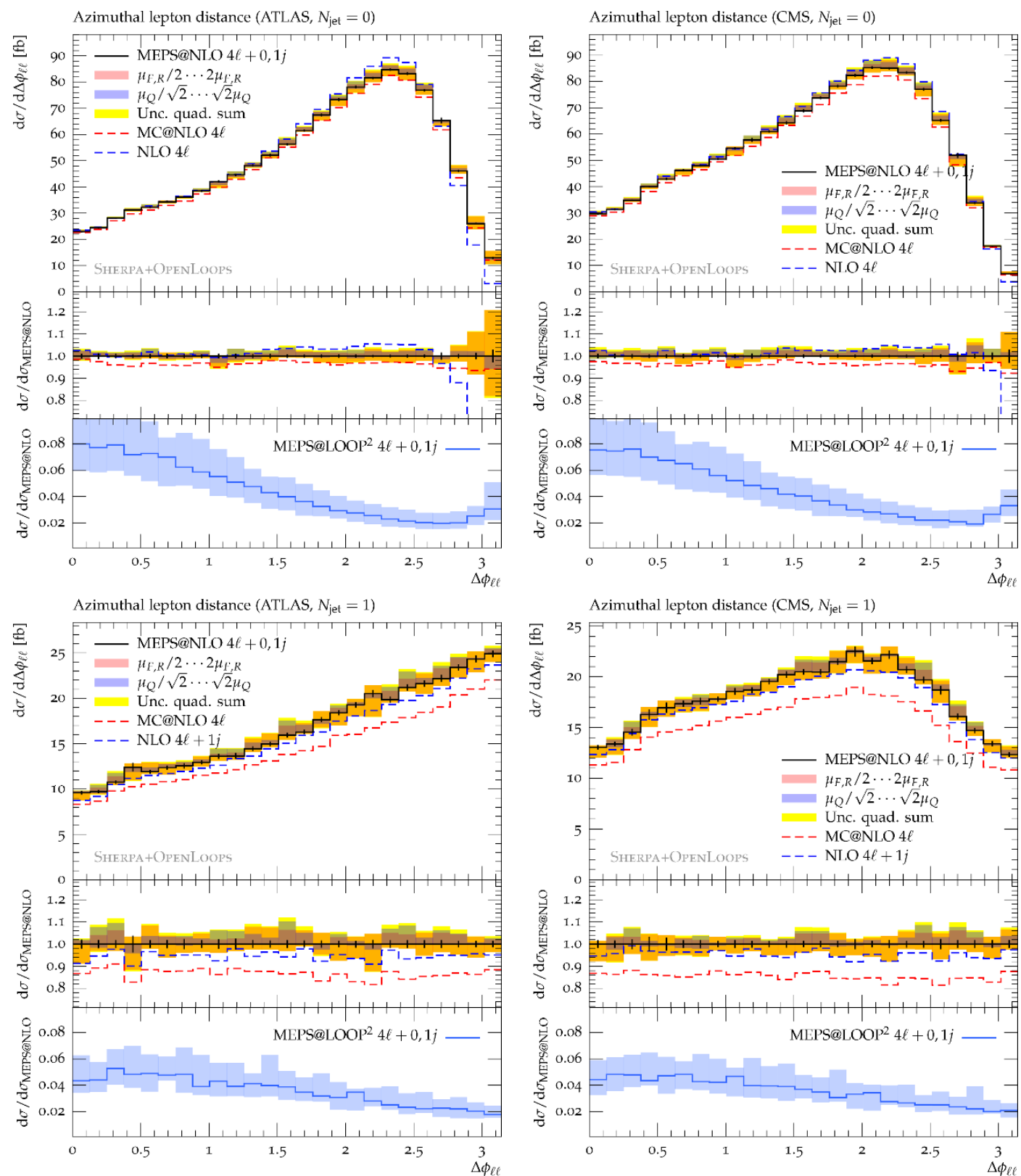

Figure 9. AtLAS (left) and Cms (right) analysis at $8 \mathrm{TeV}$ after pre-selection cuts: azimuthal separation of the charged leptons in the 0-jet (top) and 1-jet (bottom) bins. Similar predictions and uncertainty bands as in figure 8 . 

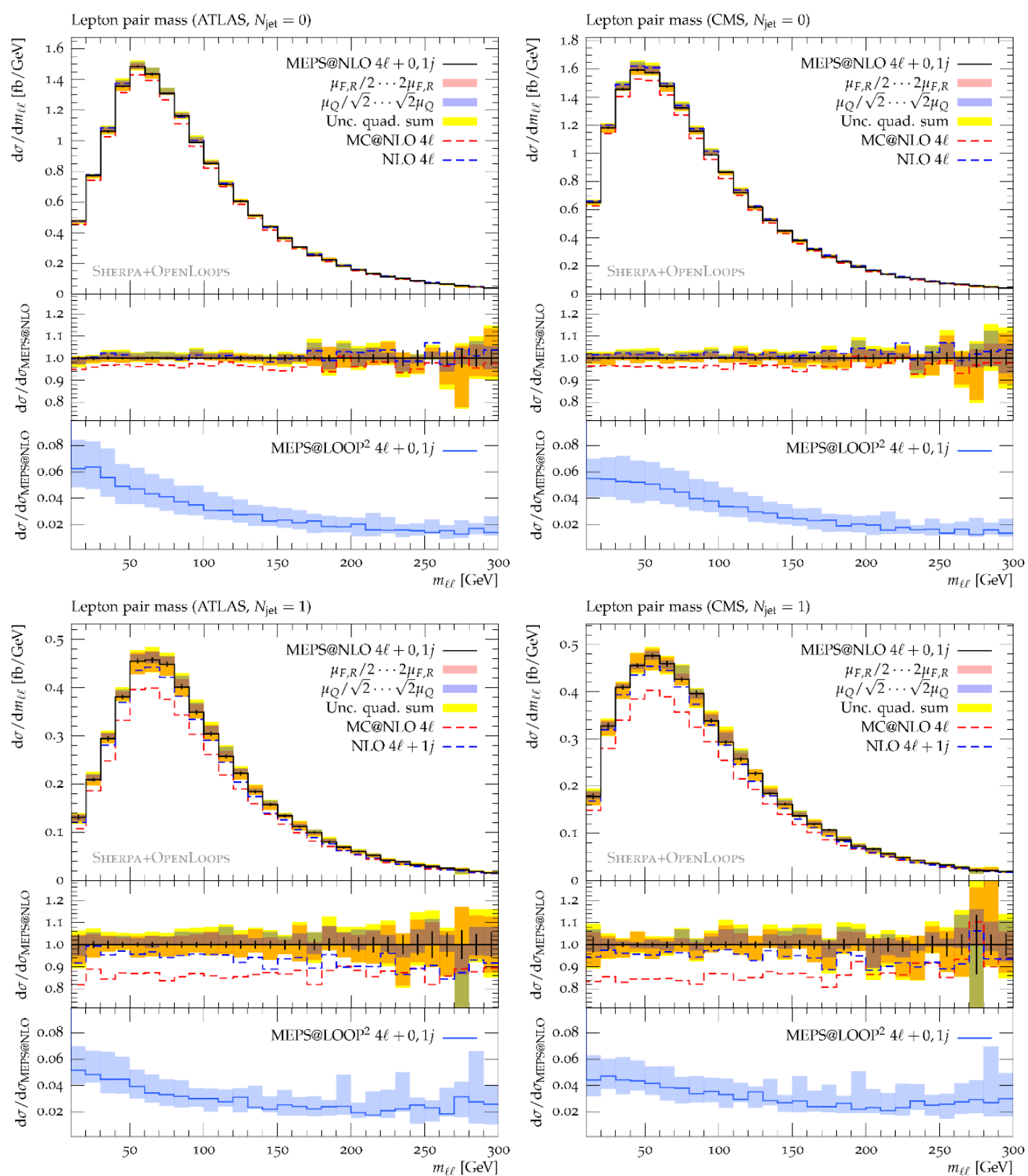

Figure 10. ATLAS (left) and CMS (right) analysis at $8 \mathrm{TeV}$ after pre-selection cuts: dilepton invariant mass distribution in the 0-jet (top) and 1-jet (bottom) bins. Similar predictions and uncertainty bands as in figure 8 . 


\begin{tabular}{|c|c|c|c|c|}
\hline 0-jet bin & NLO $4 \ell(+1 j)$ & 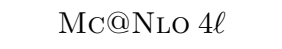 & MePs@NLO $4 \ell+0,1 j$ & MEPS@LoOP ${ }^{2} 4 \ell+0,1 j$ \\
\hline$\sigma_{\mathrm{S}}[\mathrm{fb}]$ & $34.28(9){ }_{-1.6 \%}^{+2.1 \%}$ & $32.52(8){ }_{-0.8 \%}^{+2.1 \%}+0.7 \%$ & $33.81(12){ }_{-2.2 \%}^{+1.4 \%}+2.0 \%+1.6 \%$ & $1.98(2){ }_{-16.5 \%}^{+23 \%}+20 \%$ \\
\hline$\sigma_{\mathrm{C}}[\mathrm{fb}]$ & $55.76(9){ }_{-1.7 \%}^{+2.0 \%}$ & $52.28(9)+1.4 \%+1.4 \%$ & $\begin{aligned} & 54.18(15)+1.4 \%+2.5 \%+1.7 \% \\
&-1.9 \%-0.4 \%-2.0 \%\end{aligned}$ & $2.41(2)+22 \%+27 \%$ \\
\hline 1-jet bin & $\mathrm{NLO} 4 \ell(+1 j)$ & MC@NLO $4 \ell$ & MEPS@NLO $4 \ell+0,1 j$ & MEPS@LoOP ${ }^{2} 4 \ell+0,1 j$ \\
\hline$\sigma_{\mathrm{S}}[\mathrm{fb}]$ & $8.99(4) \underset{-9.5 \%}{+4.9 \%}$ & $8.02(4)+\begin{array}{l}+8.5 \% \\
-6.4 \%\end{array}-3.1 \%$ & $\begin{array}{r}9.37(9)+2.6 \%+2.5 \%-0.1 \% \\
-2.7 \%-0.0 \%-4.1 \%\end{array}$ & $0.46(1)+40 \%+2.2 \%$ \\
\hline$\sigma_{\mathrm{C}}[\mathrm{fb}]$ & $26.50(8){ }_{-12.5 \%}^{+6.4 \%}$ & $24.58(8)+6.1 \%+1.2 \%$ & $28.32(13)+\begin{array}{l}+3.1 \%+4.1 \%+0.6 \% \\
-4.7 \%-0.0 \%-2.7 \%\end{array}$ & $0.79(1) \begin{array}{l}+33 \%+15 \% \\
-20 \%-7 \%\end{array}$ \\
\hline
\end{tabular}

Table 4. Exclusive 0- and 1-jet bin $\mu^{+} \nu_{\mu} \mathrm{e}^{-} \bar{\nu}_{\mathrm{e}}+$ jets cross sections in the signal (S) and control (C) regions of the ATLAS analysis at $8 \mathrm{TeV}$. Fixed-order NLO results (with appropriate jet multiplicity) are compared to MC@NLO and MePs@NLO predictions. Squared quark-loop contributions $\left(\right.$ MePs@Loop $\left.{ }^{2}\right)$ are presented separately. Scale uncertainties are shown as $\sigma \pm \delta_{\mathrm{QCD}} \pm \delta_{\text {res }} \pm \delta_{Q_{\text {cut }}}$, where $\delta_{\mathrm{QCD}}, \delta_{\text {res }}$ and $\delta_{Q_{\text {cut }}}$ correspond respectively to variations of the $\mathrm{QCD}\left(\mu_{\mathrm{R}}, \mu_{\mathrm{F}}\right)$, resummation $\left(\mu_{Q}\right)$ and merging $\left(Q_{\text {cut }}\right)$ scales. Statistical errors are given in parenthesis.

\begin{tabular}{|c|c|c|c|c|}
\hline 0-jet bin & NLO $4 \ell(+1 j)$ & 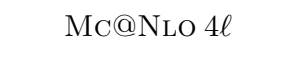 & MePs@NLO $4 \ell+0,1 j$ & Meps@Loop ${ }^{2} 4 \ell+0,1 j$ \\
\hline$\sigma_{\mathrm{S}}[\mathrm{fb}]$ & $156.65(18){ }_{-1.6 \%}^{+1.7 \%}$ & $147.8(2)+\frac{+3 \%}{-1.6 \%}+1.2 \%$ & $153.6(3){ }_{-1.9 \%}^{+2.1 \%}+2.8 \%+1.0 \%-2.2 \%$ & $6.65(4){ }_{-17 \%}^{+22 \%}+26 \%$ \\
\hline$\sigma_{\mathrm{C}}[\mathrm{fb}]$ & $59.26(15)_{-1.3 \%}^{+1.3 \%}$ & $55.92(11){ }_{-0.2 \%}^{+0.8 \%}+0.5 \%$ & $58.06(21)_{-2.0 \%}^{+2.1 \%}+2.2 \%+1.5 \%$ & $1.47(2) \begin{array}{r}+26 \%+28 \% \\
-17 \%-16 \% \\
\end{array}$ \\
\hline 1-jet bin & NLO $4 \ell(+1 j)$ & 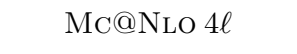 & MePs@NLO $4 \ell+0,1 j$ & MePs@Loop $4 \ell+0,1 j$ \\
\hline$\sigma_{\mathrm{S}}[\mathrm{fb}]$ & $43.01(9){ }_{-7.4 \%}^{+3.3 \%}$ & $37.87(9)+\frac{+7.6 \%}{+6.8 \%}+0.9 \%$ & $44.99(18)_{-4.2 \%}^{+2.5 \%}+2.9 \%+0.0 \%-2.5 \%$ & $1.83(2){ }_{-20 \%}^{+34 \%}+6 \%$ \\
\hline$\sigma_{\mathrm{C}}[\mathrm{fb}]$ & $20.48(6)_{-10.3 \%}^{+4.8 \%}$ & $18.90(7)_{-7.3 \%}^{+7.4 \%}+1.8 \%$ & $21.70(11)_{-4.1 \%}^{+3.2 \%}+3.0 \%+{ }_{-0.5 \%}^{+0.5 \%}$ & $0.62(1){ }_{-16 \%}^{+39 \%}+6 \%$ \\
\hline
\end{tabular}

Table 5. Exclusive 0- and 1-jet bin $\mu^{+} \nu_{\mu} \mathrm{e}^{-} \bar{\nu}_{\mathrm{e}}+$ jets cross sections in the signal (S) and control (C) regions of the CMS analysis at $8 \mathrm{TeV}$. Similar predictions and conventions as in table 4 .

largest squared quark-loop corrections arise in the 0-jet bin, at small $m_{\ell \ell^{\prime}}$ and at large $m_{\mathrm{T}}$. The corrections to the transverse-mass distribution start growing at $m_{\mathrm{T}}=100-150 \mathrm{GeV}$ and for the AtLAS (CMS) analysis they reach 10-20\%(5-10\%) in the tail. The largest effects arise in the signal region and in the ATLAS analysis, which implements tighter $m_{\ell \ell^{\prime}}$ and $\Delta \phi_{\ell \ell^{\prime}}$ cuts. For what concerns the $m_{\ell \ell^{\prime}}$ distribution, figure 13 shows that in the 0 -jet bin of the CMS signal region squared quark-loop corrections behave similarly as in the inclusive case (cf. figure 5). The fact that the characteristic enhancement at small $m_{\ell \ell^{\prime}}$ is not visible in the ATLAS signal region, is simply due to the cut on $m_{\ell \ell^{\prime}}$ at $50 \mathrm{GeV}$. For what concerns the 1-jet bin, MEPs@Loop ${ }^{2}$ corrections are generally slightly smaller and less dependent on $m_{\mathrm{T}}$ and $m_{\ell \ell^{\prime}}$.

\subsection{Exclusive 0- and 1-jet bin cross sections in control and signal regions}

A precise quantitative assessment of the various correction effects and residual uncertainties is provided in tables 4 and 5, where we present exclusive 0 - and 1-jet bin cross sections in the signal and control regions of the two experimental analyses. The NLO and MePs@NLO predictions at the central scale differ by only $1.5-3 \%$ and $4-6 \%$ in the 0 -jet and 1 -jet bins, respectively. This confirms that the discrepancy of order $5 \%$ observed in the inclusive 0 -jet 

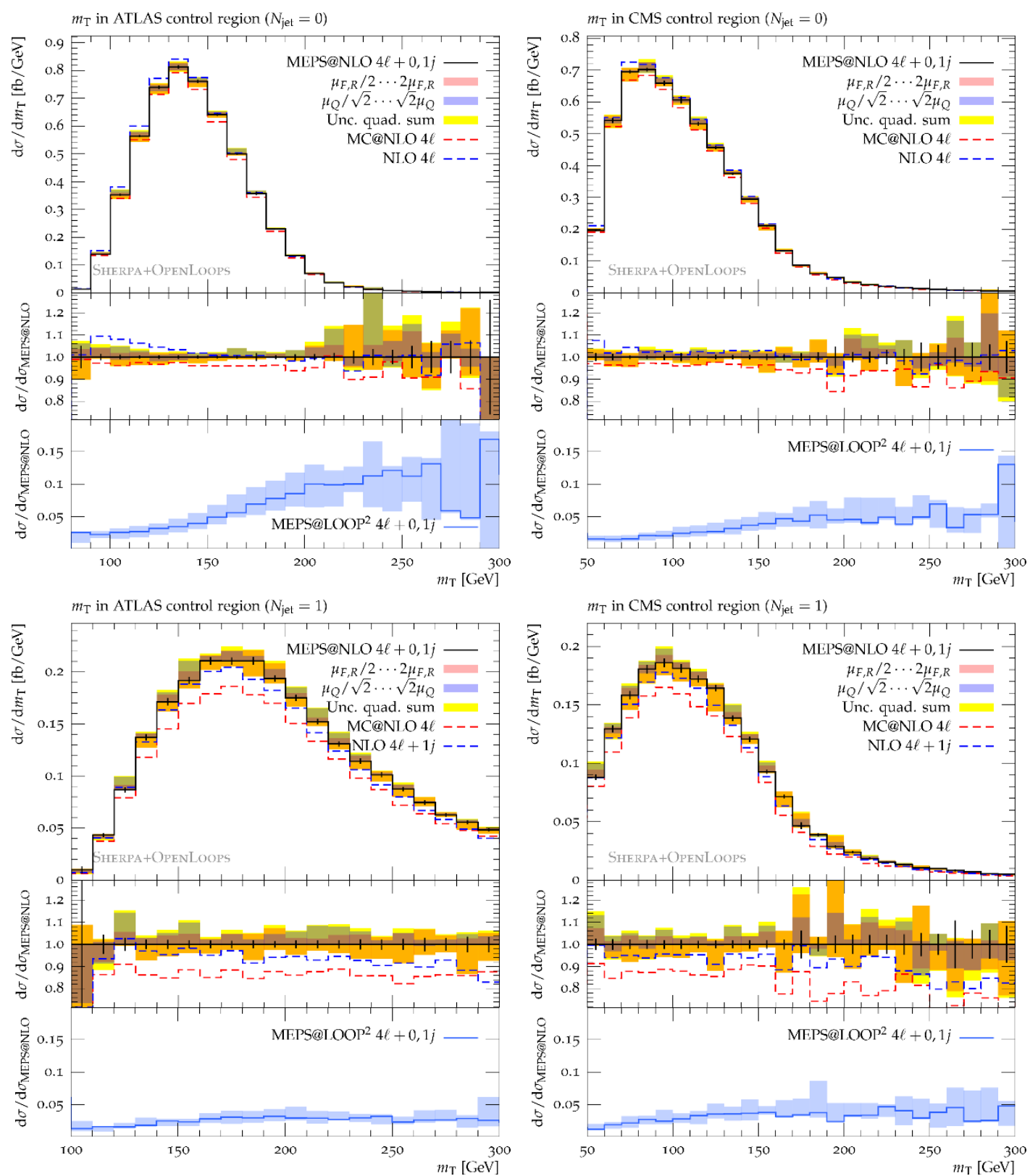

Figure 11. Control region of the ATLAS (left) and CMs (right) analysis at $8 \mathrm{TeV}$ : transverse-mass distribution in the 0-jet (top) and 1-jet (bottom) bins. Similar predictions and uncertainty bands as in figure 8 . 

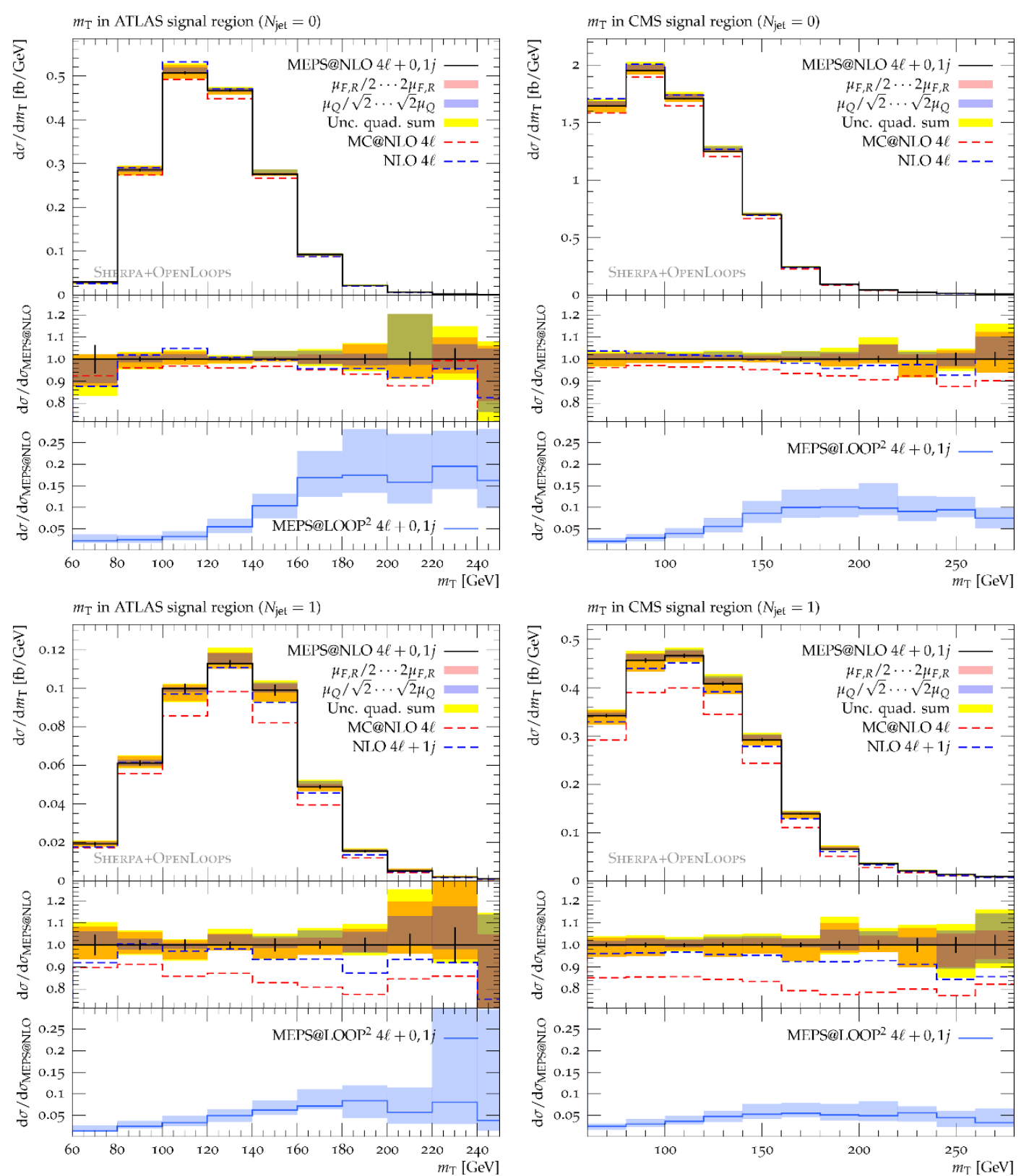

Figure 12. Signal region of the AtLAs (left) and CMs (right) analysis at $8 \mathrm{TeV}$ : transverse-mass distribution in the 0-jet (top) and 1-jet (bottom) bins. Similar predictions and uncertainty bands as in figure 8 . 

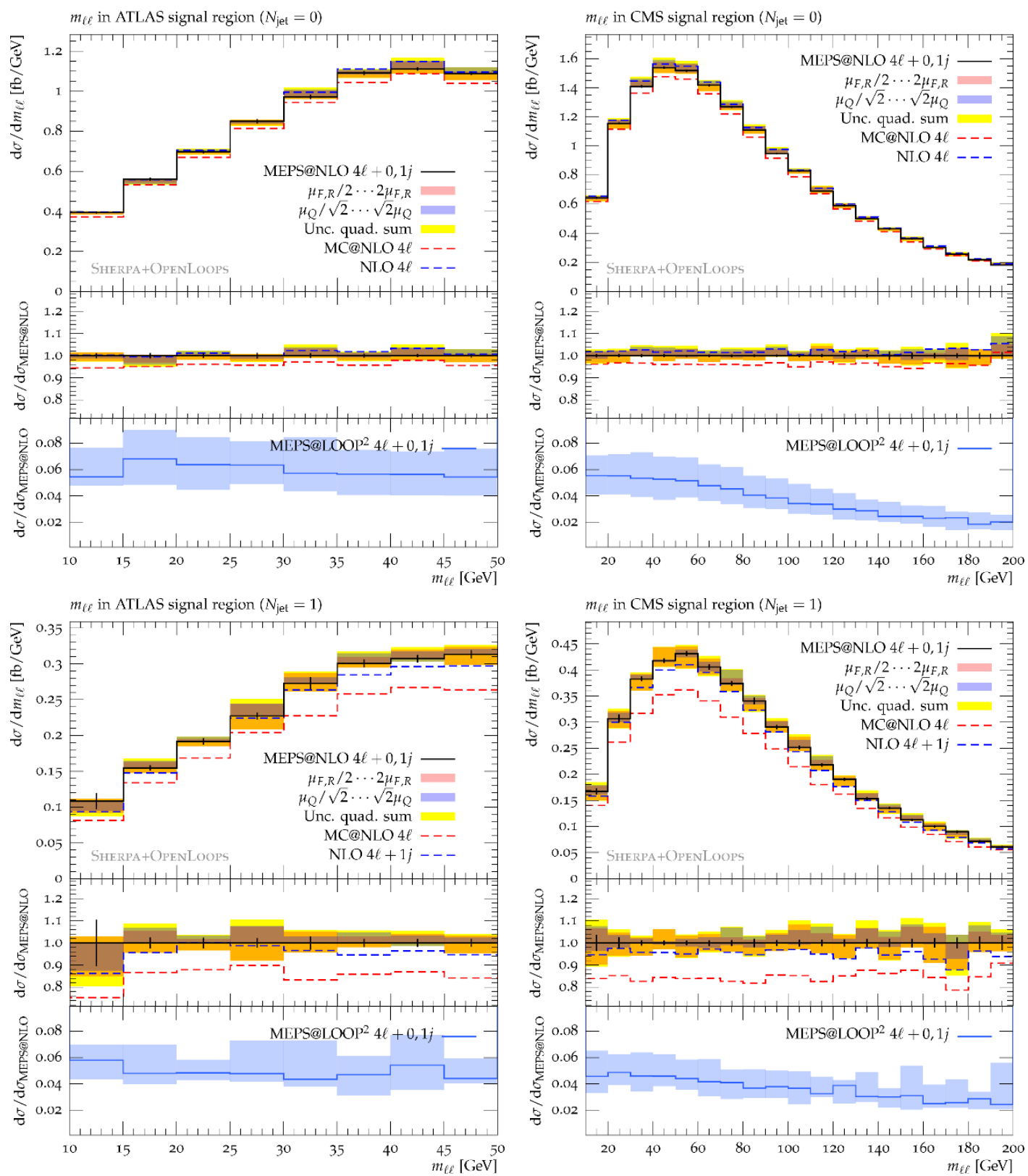

Figure 13. Signal region of the ATLAs (left) and CMS (right) analysis at $8 \mathrm{TeV}$ : dilepton invariantmass distribution in the 0-jet (top) and 1-jet (bottom) bins. Similar predictions and uncertainty bands as in figure 8 . 
bin (cf. table 2) is due the NLO corrections to the first jet emission in Meps@NLO. The differences between MC@NLO and MePs@NLO in the exclusive 0- and 1-jet bins reach $2-4 \%$ and $13-16 \%$, respectively, and the discrepancy in the 1 -jet bin is consistent with the deficit of MC@NLO observed in differential distributions. Deviations between the NLO, MC@NLO and MEPS@NLO approximations are fairly similar in the various analyses and kinematic regions. As compared to corresponding results in ref. [31], the various cross sections in tables 4 and 5 differ by 1-5\% and 1-10\% in the 0 - and 1-jet bins, respectively. These shifts are consistent with scale-variation uncertainties and can be attributed, as observed in section 4.1, to the new scale choice (3.5) used in the present study.

Adding $\left(\mu_{\mathrm{R}}, \mu_{\mathrm{F}}\right), \mu_{Q}$ and $Q_{\text {cut }}$ variations in quadrature, the combined scale uncertainties of MePs@NLO cross-section predictions do not exceed 4(6)\% in the 0(1)-jet bin. Renormalisation-, factorisation-, resummation- and merging-scale variations yield comparable contributions to the total scale uncertainty. In the 1-jet bin, MEPS@NLO results feature smaller QCD-scale variations as compared to the NLO calculation. This can be attributed to the variation of extra $\alpha_{s}$ terms originating from the shower and to the CKKW scale choice in MePs@NLO.

Comparing NLO and MC@NLO cross sections in the 0-jet bin we observe a rather constant difference of about $5 \%$ that can be interpreted as the contribution from resummed Sudakov logarithms beyond NLO. On the one hand, this indicates that matching to the parton shower is essential in order to reach few-percent precision. On the other hand, the rather mild impact of Sudakov resummation suggests that subleading Sudakov logarithms beyond the shower approximation should not have a large impact on the $\mathrm{H} \rightarrow \mathrm{WW}^{*}$ analysis. This is confirmed by the fact that resummation-scale variations of MC@NLO and MePs@NLO cross sections do not exceed 2-3\% in the various jet bins.

The relative impact of squared quark-loop corrections as compared to merged NLO predictions varies between 2.5 and 6 percent, depending on the experiment, the kinematic selection region, and the jet bin. In both experiments and jet bins, squared quark-loop effects increase when moving from control to signal regions. In the case of CMS they grow from $2-3.5 \%$ to $4 \%$, while in the AtLAs analysis, due to the tighter $\Delta \phi_{\ell \ell^{\prime}}$ and $m_{\ell \ell^{\prime}}$ cuts, the effects are more pronounced and increase from $3-4.5 \%$ to $5-6 \%$. Squared quark-loop uncertainties amount to 30-40\%, similarly as for the inclusive analysis of section 4 .

Detailed results for the ratios of signal- to control-region cross sections, $\sigma_{\mathrm{S}} / \sigma_{\mathrm{C}}$, are presented in table 6 . These ratios and the related uncertainties play an important role for the extrapolation from control to signal regions in data-driven WW-background determinations. In addition to NLO, MC@NLO and MePs@NLO ratios, we also present results obtained from the combination of NLO and squared quark-loop merging. These latter are denoted as MEPs@NLO+LOOP ${ }^{2}$ and represent our best predictions. Upper and lower variations are obtained from corresponding QCD-, resummation- and merging-scale variations in tables 4 and 5. More precisely, the ratios are evaluated at different scales,

$$
R\left(\xi_{\mathrm{R}}, \xi_{\mathrm{F}}, \xi_{Q}, Q_{\text {cut }}\right)=\frac{\sigma_{\mathrm{S}}\left(\xi_{\mathrm{R}} \mu_{\mathrm{R}}, \xi_{\mathrm{F}} \mu_{\mathrm{F}}, \xi_{Q} \mu_{Q}, Q_{\mathrm{cut}}\right)}{\sigma_{\mathrm{C}}\left(\xi_{\mathrm{R}} \mu_{\mathrm{R}}, \xi_{\mathrm{F}} \mu_{\mathrm{F}}, \xi_{Q} \mu_{Q}, Q_{\text {cut }}\right)},
$$

applying correlated variations in signal and control regions. As shown in table 6 , due to 


\begin{tabular}{|c|c|c|c|c|c|}
\hline AtLas & NLO $4 \ell(+1 j)$ & MC@NLO $4 \ell$ & MePs@NLO $4 \ell+0,1 j$ & MEPS@NLO+LoOP ${ }^{2}$ & $\delta_{\mathrm{S} / \mathrm{C}}$ \\
\hline$\sigma_{\mathrm{S}} / \sigma_{\mathrm{C}} 0$-jet & $0.615_{-0.1 \%}^{-0.1 \%}$ & $0.622-0.7 \%+0.2 \%$ & $0.624{ }_{-0.3 \%}^{+0 \%}{ }_{-0 \%}^{+0.5 \%}{ }_{-0.3 \%}^{+0.1 \%}$ & $\begin{array}{l}0.632-0.3 \%+0.2 \% \\
+0.5 \%+0.3 \%\end{array}$ & $1.3 \%$ \\
\hline$\sigma_{\mathrm{S}} / \sigma_{\mathrm{C}} 1$-jet & $0.339_{-3.4 \%}^{+1.4 \%}$ & $\begin{aligned} 0.326_{-0.1 \%}^{-2.3 \%}+1.2 \% \\
-0.1 \% \\
\end{aligned}$ & 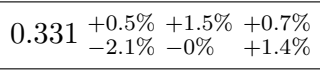 & $\begin{array}{r}0.338-0.4 \%+1.8 \% \\
-1.8 \%+0.1 \% \\
\end{array}$ & $2.1 \%$ \\
\hline CMS & NLO $4 \ell(+1 j)$ & MC@NLO $4 \ell$ & MEPS@NLO $4 \ell+0,1 j$ & MEPS@NLO+LoOP ${ }^{2}$ & $\delta_{\mathrm{S} / \mathrm{C}}$ \\
\hline$\sigma_{\mathrm{S}} / \sigma_{\mathrm{C}} 0$-jet & $2.64_{+0.3 \%}^{-0.4 \%}$ & 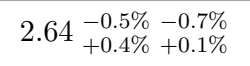 & $2.65{ }_{-0.1 \%}^{+0 \%}-0.6 \%-0.1 \%$ & 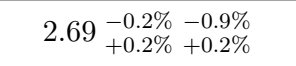 & $1.5 \%$ \\
\hline$\sigma_{\mathrm{S}} / \sigma_{\mathrm{C}} 1$-jet & $2.10_{-3.2 \%}^{+1.4 \%}$ & $2.00 \begin{array}{l}-0.2 \% \\
-0.5 \%+0.9 \% \\
\end{array}$ & $2.07 \begin{array}{c}+0.7 \% \\
+0.1 \%-0.5 \%\end{array}+\stackrel{+0 \%}{+0.9 \%}$ & $2.10 \begin{array}{l}+0.4 \%+0.7 \% \\
+0.4 \%+0.1 \%\end{array}$ & $1.4 \%$ \\
\hline
\end{tabular}

Table 6. Ratios of signal- to control-region cross sections in the 0- and 1-jet bins of the two experimental analyses. Fixed-order NLO results (with appropriate jet multiplicity) are compared to MC@Nlo and MePs@Nlo predictions. The combination of NLO and squared quark-loop merged results, denoted as MEPS@NLO+LOOP ${ }^{2}$, represents the best prediction. Upper and lower variations are obtained from corresponding QCD-, resummation- and merging-scale uncertainties in tables 4 and 5 assuming correlated $\sigma_{\mathrm{S}}$ and $\sigma_{\mathrm{C}}$ variations. The last column shows the relative difference between MEPs@NLO and full MEPs@NLO+LoOP ${ }^{2}$ predictions, which corresponds to the shift induced by squared quark-loop corrections.

almost complete cancellations between $\sigma_{\mathrm{S}}$ and $\sigma_{\mathrm{C}}$ variations this naive approach results in typical $\sigma_{\mathrm{S}} / \sigma_{\mathrm{C}}$ shifts at the sub-percent level, which cannot be regarded as realistic estimates of uncertainties due to unknown higher-order corrections. On the other hand, applying uncorrelated scale variations to $\sigma_{\mathrm{S}}$ and $\sigma_{\mathrm{C}}$ would tend to overestimate $\sigma_{\mathrm{S}} / \sigma_{\mathrm{C}}$ uncertainties. This becomes clear if one considers the ideal limit of identical signal and control regions, where $\sigma_{\mathrm{S}} / \sigma_{\mathrm{C}}=1$ and the uncertainty must vanish. The reason why scale variations are not adequate to quantify theory uncertainties associated to the extrapolation between different kinematic regions, is that they tend to shift the normalisation of scattering amplitudes without altering their kinematic dependence. In this respect, squared quarkloop corrections provide much more useful insights into kinematic effects associated to higher-order corrections. As shown in the last column of table 6 , their impact on the $\sigma_{\mathrm{S}} / \sigma_{\mathrm{C}}$ ratios amounts to $\delta_{\mathrm{S} / \mathrm{C}} \simeq 1.5 \%$, which largely exceeds the typical scale variations of MePs@NLO and MePs@NLO+LOOP ${ }^{2}$ predictions. This is due to the fact that squared quark-loop effects induce genuine NNLO kinematic distortions. Moreover, squared quark loops constitute only a subset of the full NNLO corrections, and their impact on $\sigma_{\mathrm{S}} / \sigma_{\mathrm{C}}$ can be assumed to be quantitatively similar to the still unknown NNLO contributions. With other words, the $\delta_{\mathrm{S} / \mathrm{C}}$ shifts in table 6 can be considered as a realistic estimate of the MePs@NLO+LoOP ${ }^{2}$ uncertainty of the $\sigma_{\mathrm{S}} / \sigma_{\mathrm{C}}$ ratios.

\section{Conclusions}

In this publication we have presented the first results for the simulation of hadronic fourlepton plus jets production using the novel MEPs@NLO multi-jet merging technology at NLO, and including also NNLO contributions from squared quark loops. This was also the first phenomenological application of the fully automated approach provided by the combination of the SHERPA Monte Carlo with the OpenLoops generator of one-loop amplitudes. 
The OpenLoops algorithm is based on a new numerical approach for the recursive construction of cut-opened loop diagrams, which allows for a very fast evaluation of NLO matrix elements within the Standard Model. For the calculation of tensor integrals it relies on the ColLiER library, which implements the numerically stable reduction algorithms by Denner-Dittmaier.

Four-lepton plus jets final states are of large topical interest due to their implications on ongoing Higgs-boson studies, and in this paper we discussed detailed predictions for the Atlas and Cms $\mathrm{H} \rightarrow \mathrm{WW}^{*}$ analyses at $8 \mathrm{TeV}$ in the 0 - and 1-jet bins. For a thorough description of four-lepton production - including off-shell vector-boson effects, non-resonant topologies, and related interferences - the complex-mass scheme was applied. The use of exclusive jet bins, which is mandatory in order to suppress the background provided by top-quark production and decay, introduces potentially large theory uncertainties and ultimately requires a very robust modelling of jet-production properties and related errors. This requires an NLO accurate description of jet radiation, with a careful assessment of the uncertainties stemming from the usual perturbative scale variations, but also a resummation of Sudakov logarithms arising from jet vetoes, and an analysis of the related uncertainties. The MePs@Nlo approach as implemented in SHERPA allows to carry out this program in a fully automated way. In particular, the resummation of Sudakov logarithms is effectively implemented by matching NLO matrix elements to the SHERPA parton shower, and uncertainties related to subleading Sudakov logarithms beyond the shower approximation can be assessed through resummation-scale variations.

In order to allow precise statements on the impact of jet vetoes and jet binning on the $\mathrm{H} \rightarrow \mathrm{WW}^{*}$ analyses, we merged matrix elements for four leptons plus up to one jet at NLO accuracy, thus arriving at a simulation of the WW background with unprecedented accuracy. As a result of this calculation the residual scale uncertainty is reduced to about $5 \%$ on observables related to the hardest jet up to transverse momenta of the order of $200 \mathrm{GeV}$. We note large differences of up to $40 \%$ with respect to NLO or MC@NLO simulations of the pp $\rightarrow 4 \ell$ process. These differences typically manifest themselves in regions of large jet momentum, where inclusive NLO or MC@NLO predictions are bound to undershoot the QCD activity. This of course is even more pronounced for observables related to the subleading jet. As compared to NLO predictions for $\mathrm{pp} \rightarrow 4 \ell+1 j$, apart from a generally good agreement, multi-jet merging yields quite significant corrections in the tail of the first-jet $p_{\mathrm{T}}$ distribution. This effect can be attributed to the fact that the CKKW-merging approach implemented in MEPS@NLO consistently adapts the renormalisation scale to the transverse momenta of the emitted jets.

The multi-jet merging thus improves the quality and stability of the perturbative series, especially for jet observables. This holds for hard phase-space regions as well as for low jet momentum, where fixed-order calculations start to suffer from the missing resummation of potentially large logarithms. Studying the case of a jet veto, we found that for veto scales around 30(10) GeV resummation effects beyond NLO amount to about 5(20)\% of the vetoed four-lepton cross section. Their relatively small magnitude can be attributed to the limited size of Sudakov logarithms but also to cancellations between leading- and subleading-logarithmic contributions. 
In the case of the inclusive four-lepton cross section, as a result of NLO corrections to the first QCD emission, MEPS@NLO results turn out to be $9 \%$ higher as compared to inclusive NLO and MC@NLO calculations. Moreover, the CKKW scale choice in MEPS@NLO leads to a milder renormalisation-scale dependence as compared to fixed-order and Mc@NLO predictions evaluated at a scale of the order of the W-boson transverse mass. For leptonic observables in the exclusive jet bins of the $\mathrm{H} \rightarrow \mathrm{WW}^{*}$ analyses, typically NLO and MC@NLO provide a good description in the 0-jet bin, but MC@NLO exhibits a deficit of about $10-15 \%$ in the 1-jet bin. It is notable that, for these observables, we find scale uncertainties of only a few percent in our best NLO prediction, i.e. MEPs@NLO. Our analysis indicates that also the uncertainties related to the choice of resummation scale, and thus due to the parton shower and its resummation properties, are at the percent level. This is consistent with the observation that Sudakov logarithms beyond NLO have a rather moderate impact on the jet bins of the $\mathrm{H} \rightarrow \mathrm{WW}^{*}$ analysis, and it suggests that subleading logarithmic corrections beyond the MEPS@NLO accuracy should not be important.

In addition to matched and merged NLO simulations, we also studied NNLO contributions to four-lepton plus jets production that emerge through squared one-loop amplitudes involving closed quark loops. These contributions are dominated by the gluon-gluon channel, which is enhanced by the high partonic flux. Moreover, squared quark-loop corrections are quite sensitive to lepton-lepton correlations that play a key role in the $\mathrm{H} \rightarrow \mathrm{WW}^{*}$ analysis. Their relative impact as compared to the full NLO contributions amounts to only $3 \%$ in the inclusive case, but grows to $6 \%$ if Higgs-analysis cuts are applied. This corresponds to about $50 \%$ of the Higgs-boson signal in the relevant analysis regions, which calls for a detailed theoretical investigation of squared quark-loop terms and of their nontrivial kinematic features. To this end we considered all relevant squared quark-loop matrix elements for the production of four leptons plus up to one jet. In particular, in addition to the well-known gluon-gluon fusion contributions, for the first time we also studied the $\mathrm{g} q \rightarrow 4 \ell+q, \mathrm{~g} \bar{q} \rightarrow 4 \ell+\bar{q}$, and $q \bar{q} \rightarrow 4 \ell+\mathrm{g}$ channels. In order to merge squared quark-loop corrections with different jet multiplicity, we extended the tree-level multi-jet merging in SHERPA to include also purely loop-induced processes. In this context, the inclusion of the quark channels is indispensable for a consistent merging. The net effect of this merging is a visibly harder tail in the jet transverse momentum distribution with respect to the one obtained from only taking the leading gg $\rightarrow 4 \ell$ contribution supplemented with the parton shower. To the best of our knowledge this has not been studied before.

In the $\mathrm{H} \rightarrow \mathrm{WW}^{*}$ analyses, the size of squared quark-loop corrections turns out to vary from $2 \%$ to $6 \%$, depending on the jet bin, on the kinematic region and on the experiment. The merging approach is especially important in order to guarantee decent predictions in the 1-jet bin. Due to their nontrivial kinematic dependence, squared quark-loop corrections have a quite significant impact on the extrapolation of the WW-background from control to signal regions. The resulting shift in the relevant cross-section ratios is of order $1.5 \%$, and we argued that these corrections can be regarded as a realistic estimate of unknown higher-order effects in the data-driven determination of the WW-background at the LHC.

At this point it should be stressed that all the studies reported here are at the parton level only, with one choice of PDFs to facilitate a clear and direct comparison between the 
different approaches. It is, however, a straightforward exercise to allow for different PDFs or to go from the parton to the hadron level in a simulation like the one presented here: switching on hadronisation and the underlying event modelling allows to assess these effects automatically. As a further extension, it is possible to extend the current study to cases including all possible other four-lepton final states or to study in more detail the two-jet bin of the simulation, which is crucial for the vector-boson fusion signatures. For the latter case, the simulation could be extended to the production of four leptons in association with two jets at next-to leading order accuracy. It can be anticipated that a simulation on the level presented here would certainly lead to a similarly relevant reduction of QCD uncertainties for this important channel of Higgs physics.

\section{A Treatment of bottom- and top-quark contributions}

Consistently with the five-flavour evolution of PDFs and $\alpha_{s}$, for bottom quarks we adopt the massless approximation. Top quarks are thus the only QCD partons that we treat as massive. They can contribute to $\mathrm{pp} \rightarrow \mathrm{W}^{+} \mathrm{W}^{-}+$jets through closed quark loops, but also via resonant top propagators in sub-processes with external b quarks, such as gb $\rightarrow$ $\mathrm{W}^{+} \mathrm{W}^{-} \mathrm{b}$ and $\mathrm{gg} \rightarrow \mathrm{W}^{+} \mathrm{W}^{-} \mathrm{b} \overline{\mathrm{b}}$. Partonic channels of this type are dominated by $\mathrm{Wt}$ and $t \overline{\mathrm{t}}$ production, and are more conveniently handled as separate processes. Therefore, as operational definition of $\mathrm{W}^{+} \mathrm{W}^{-}+$jets production, we consider only partonic channels that do not involve $b$ quarks in the initial or final state. As pointed out in ref. [17], when excluding external b quarks, care must be taken to avoid NLO infrared singularities in $\mathrm{pp} \rightarrow \mathrm{W}^{+} \mathrm{W}^{-} j$. This issue is related to the renormalisation of the external-gluon wave function, which receives a b-quark contribution

$$
\delta Z_{A}^{(\mathrm{b})}=\frac{\alpha_{s}}{6 \pi}\left[\mu^{2 \varepsilon} \Delta_{\mathrm{IR}}-\mu^{2 \varepsilon} \Delta_{\mathrm{UV}}\right]=0,
$$

where $\mu$ is the scale of dimensional regularisation, and infrared (IR) and ultraviolet (UV) singularities in $D=4-2 \varepsilon$ dimensions yield

$$
\Delta_{\mathrm{IR}, \mathrm{UV}}=\frac{(4 \pi)^{\varepsilon}}{\Gamma(1-\varepsilon)} \frac{1}{\varepsilon}, \quad \mu^{2 \varepsilon} \Delta_{\mathrm{IR}, \mathrm{UV}}=\Delta_{\mathrm{IR}, \mathrm{UV}}+\ln \mu^{2}+\mathcal{O}(\varepsilon) .
$$

The renormalisation constant (A.1) vanishes due to an exact IR-UV compensation. However, while its UV pole $\mu^{2 \varepsilon} \Delta_{\mathrm{UV}}$ cancels in renormalised $q \bar{q} \rightarrow \mathrm{W}^{+} \mathrm{W}^{-} \mathrm{g}$ amplitudes, ${ }^{7}$ the compensation of the IR pole $\mu^{2 \varepsilon} \Delta_{\mathrm{IR}}$ requires a $q \bar{q} \rightarrow \mathrm{W}^{+} \mathrm{W}^{-} \mathrm{b} \overline{\mathrm{b}}$ real-emission counterpart involving collinear $\mathrm{g} \rightarrow \mathrm{b} \overline{\mathrm{b}}$ splittings. The inclusion of $\mathrm{W}^{+} \mathrm{W}^{-} \mathrm{b} \overline{\mathrm{b}}$ final states - at least in the collinear region - is thus indispensable for an infrared-safe NLO definition of $\mathrm{W}^{+} \mathrm{W}^{-} j$ production in the five-flavour scheme.

In ref. [17], the IR cancellation was achieved by including the contribution of $\mathrm{g} \rightarrow \mathrm{b} \overline{\mathrm{b}}$ splittings to the Catani-Seymour $\mathcal{I}$-operator [53],

$$
\mathcal{I}^{(\mathrm{b})}=-\frac{\alpha_{s}}{6 \pi}\left[\Delta_{\mathrm{IR}}+\frac{1}{2}\left(\ln \frac{\mu^{2}}{2 p_{q} p_{\mathrm{g}}}+\ln \frac{\mu^{2}}{2 p_{\bar{q}} p_{g}}\right)+\frac{8}{3}\right],
$$

\footnotetext{
${ }^{7}$ Here we discuss only partonic processes with gluons in the final state. Similar arguments apply also to the crossing-related $q \mathrm{~g} \rightarrow \mathrm{W}^{+} \mathrm{W}^{-} q$ and $\bar{q} \mathrm{~g} \rightarrow \mathrm{W}^{+} \mathrm{W}^{-} \bar{q}$ channels.
} 
where $p_{q}, p_{\bar{q}}$ and $p_{\mathrm{g}}$ are the quark, anti-quark and gluon momenta, respectively. Combining $\delta Z_{A}^{(\mathrm{b})}+\mathcal{I}^{(\mathrm{b})}$ yields an IR-finite and $\ln \mu$-independent result. The $\mathcal{I}$-operator contribution (A.3) results from dipole-subtraction terms, which approximate $\mathrm{g} \rightarrow \mathrm{b} \overline{\mathrm{b}}$ splittings in the collinear limit, upon integration over the entire $b \bar{b}$ phase space. In principle, it should be combined with a subtracted real-emission counterpart, which is free from singularities but depends on the cuts applied to the b $\bar{b}$ pair. In ref. [17], this finite real-emission part

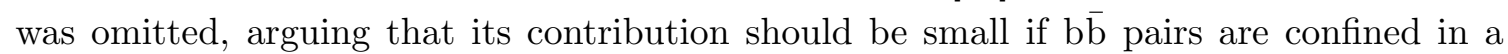
jet cone. This kinematic restriction of the $b \bar{b}$ phase space would also suppress $t \bar{t}$ and $t W$ contributions. However, confining b $\bar{b}$ pairs in narrow jets would introduce potentially large logarithms of the jet radius. Moreover, the consistent inclusion of the real-emission part would exactly cancel the $8 / 3$ term in (A.3), which results from the unphysical dipoles, and replace it by an unknown cut-dependent contribution. The inclusion of $\mathcal{I}$-operator terms (A.3) without corresponding real-emission parts should thus be regarded as a regularisation prescription, which guarantees the correct cancellation of poles and large logarithms corresponding to inclusive $\mathrm{b} \overline{\mathrm{b}}$ emission, but involves ad-hoc constant parts. This ambiguity can be removed only upon inclusion of the dipole-subtracted $\mathrm{W}^{+} \mathrm{W}^{-} \mathrm{b} \overline{\mathrm{b}}$ remnant.

Based on these considerations, we adopt a splitting approach similar to ref. [17], but we prefer to subtract only the singular and logarithmically-enhanced terms arising from inclusive $\mathrm{g} \rightarrow \mathrm{b} \overline{\mathrm{b}}$ emissions. More precisely, instead of the subtraction term (A.3) we use ${ }^{8}$

$$
\mathcal{I}_{\text {mod }}^{(\mathrm{b})}=-\frac{\alpha_{s}}{6 \pi}\left(\Delta_{\mathrm{IR}}+\ln \frac{\mu^{2}}{\mu_{\mathrm{R}}^{2}}\right) .
$$

Since the renormalisation scale $\mu_{\mathrm{R}}$ is typically of the same order of the kinematic invariants in (A.3), the main difference between (A.3) and (A.4) amounts to

$$
\mathcal{I}^{(\mathrm{b})}-\mathcal{I}_{\text {mod }}^{(\mathrm{b})}=-\frac{\alpha_{s}}{6 \pi}\left[\frac{1}{2}\left(\ln \frac{\mu_{\mathrm{R}}^{2}}{2 p_{q} p_{\mathrm{g}}}+\ln \frac{\mu_{\mathrm{R}}^{2}}{2 p_{\bar{q}} p_{g}}\right)+\frac{8}{3}\right] \simeq-\frac{4 \alpha_{s}}{9 \pi} \simeq-1.7 \%,
$$

and can be regarded as the typical ambiguity inherent in the separation of the $\mathrm{W}^{+} \mathrm{W}^{-} j$ and $\mathrm{W}^{+} \mathrm{W}^{-} \mathrm{b} \overline{\mathrm{b}}$ cross sections. Note that, in order to reflect this kind of uncertainty in standard scale-variation studies, we intentionally introduce a fake $\ln \mu_{\mathrm{R}}$ dependence in the IR-subtraction term (A.4).

This small ambiguity is due to the absence of the dipole-subtracted $\mathrm{W}^{+} \mathrm{W}^{-} \mathrm{b} \overline{\mathrm{b}}$ emission, which is supposed to be included in a separate calculation of $\mathrm{W}^{+} \mathrm{W}^{-} \mathrm{b} \overline{\mathrm{b}}$ production, i.e. of $t \bar{t}$ and $\mathrm{Wt}$ off-shell production. It can be removed by combining the $\mathrm{W}^{+} \mathrm{W}^{-}+$jets and $\mathrm{W}^{+} \mathrm{W}^{-} \mathrm{b} \overline{\mathrm{b}}$ calculations in a single simulation. For a consistent matching of the two processes, the $\mathcal{I}$-operator term (A.3) in the pp $\rightarrow \mathrm{W}^{+} \mathrm{W}^{-} \mathrm{b} \overline{\mathrm{b}}$ calculation should be replaced by the finite $\operatorname{shift}^{9}$ (A.5).

In summary, due to collinear $\mathrm{g} \rightarrow \mathrm{b} \overline{\mathrm{b}}$ singularities, the splitting of $\mathrm{pp} \rightarrow \mathrm{W}^{+} \mathrm{W}^{-} j$ and $\mathrm{pp} \rightarrow \mathrm{W}^{+} \mathrm{W}^{-} \mathrm{b} \overline{\mathrm{b}}$ is not unique, and the subtraction term (A.4) corresponds to a natural matching prescription, which is free from large logarithms and ad-hoc constants.

\footnotetext{
${ }^{8}$ Technically, we circumvent the explicit implementation of the subtraction term (A.4) by assigning the values $\Delta_{\mathrm{IR}} \rightarrow 0$ and $\mu \rightarrow \mu_{\mathrm{R}}$ to the dimensional-regularisation parameters.

${ }^{9}$ Here we assume that $\mathrm{pp} \rightarrow \mathrm{W}^{+} \mathrm{W}^{-} \mathrm{b} \overline{\mathrm{b}}$ is computed using dipole subtraction, but the matching procedure can be obviously adapted to any other subtraction method.
} 


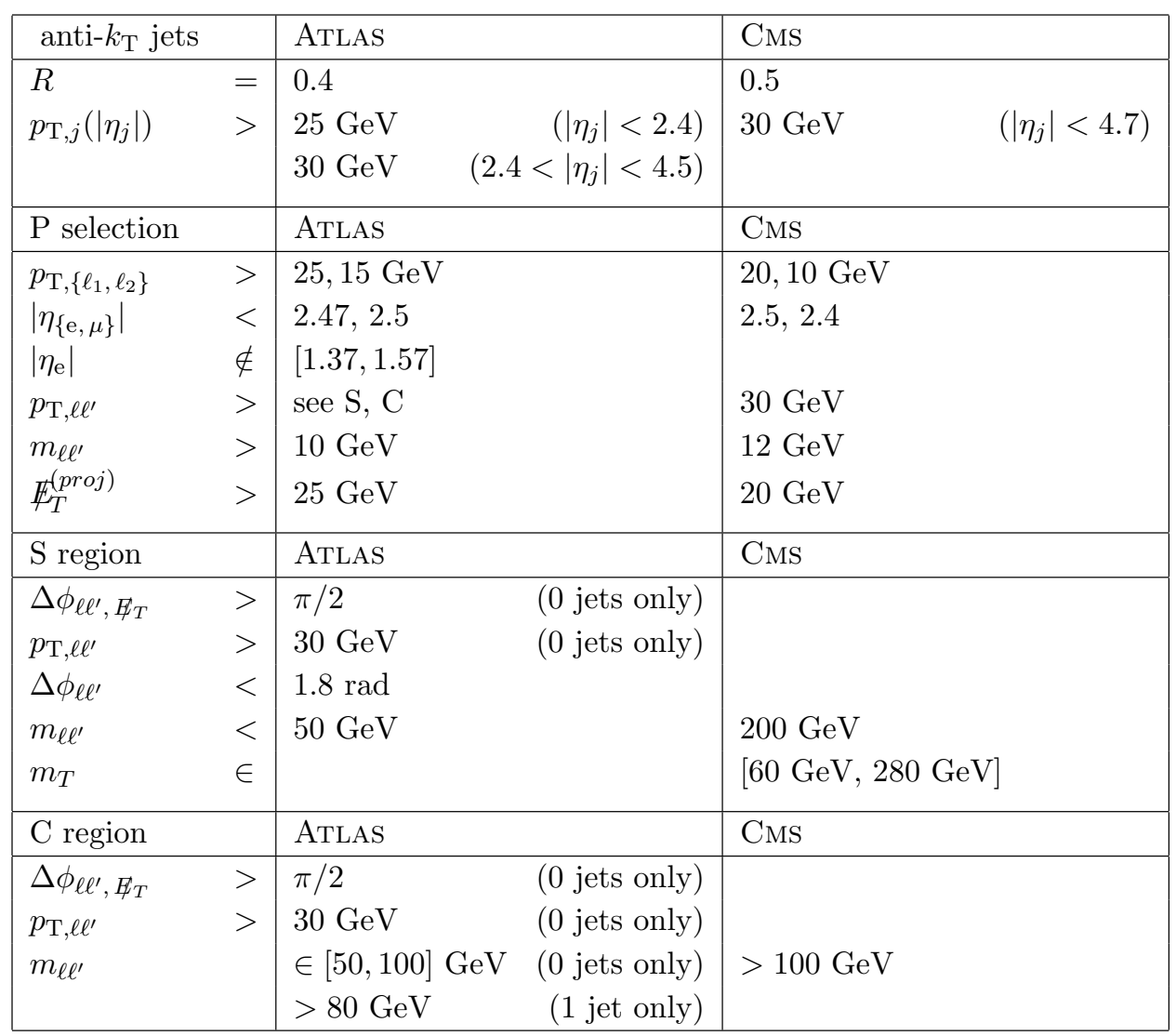

Table 7. Jet definitions and selection cuts in the AtLas and CMs analyses of $\mathrm{H} \rightarrow \mathrm{WW}^{*} \rightarrow$ $\mu^{+} \nu_{\mu} \mathrm{e}^{-} \bar{\nu}_{\mathrm{e}}$ at $8 \mathrm{TeV}$. The cuts refer to various levels and regions, namely event pre-selection ( $\mathrm{P}$ cuts), the signal region ( $\mathrm{P}$ and $\mathrm{S}$ cuts) and the control region ( $\mathrm{P}$ and $\mathrm{C}$ cuts). The projected missing transverse energy $\not_{T}^{(p r o j)}$ is defined as $\not_{\mathrm{T}}^{(\text {proj })}=\not_{\mathrm{T}} \cdot \sin \left(\min \left\{\Delta \phi_{\text {near }}, \pi / 2\right\}\right)$, where $\Delta \phi_{\text {near }}$ denotes the angle between the missing transverse momentum $\not_{\mathrm{T}}$ and the nearest lepton in the transverse plane.

\section{B Cuts of the Atras and CMs $\mathrm{H} \rightarrow \mathrm{WW}^{*}$ analyses in 0- and 1-jet bins}

The cuts of the AtLas [3] and CMS [4] $\mathrm{H} \rightarrow \mathrm{WW}^{*} \rightarrow \mu^{+} \nu_{\mu} \mathrm{e}^{-} \bar{\nu}_{\mathrm{e}}$ analyses at $8 \mathrm{TeV}$ in the exclusive 0 - and 1-jet bins are listed in table 7 . To be close to the experimental definitions of both AtLas and CMs, lepton isolation is implemented at the particle level. The scalar sum of the transverse momenta of all visible particles within a $R=0.3$ cone around the lepton candidate is not allowed to exceed $15 \%$ of the lepton $p_{\mathrm{T}}$. Partons are recombined into jets using the anti- $k_{\mathrm{T}}$ algorithm [67]. The different WW transverse-mass definition employed in ATLAS and CMS is consistently taken into account,

$$
m_{\mathrm{T}}^{2}=\left\{\begin{array}{ll}
\left(\sqrt{p_{\mathrm{T}, \ell \ell^{\prime}}^{2}+m_{\ell \ell^{\prime}}^{2}}+\not_{\mathrm{T}}\right)^{2}-\left|p_{\mathrm{T}, \ell \ell^{\prime}}+E_{\mathrm{T}}\right|^{2} & \text { for ATLAS } \\
2\left|p_{\mathrm{T}, \ell \ell^{\prime}}\right|\left|E_{\mathrm{T}}\right|\left(1-\cos \Delta \phi_{\ell \ell^{\prime}, E_{\mathrm{T}}}\right) & \text { for CMS }
\end{array},\right.
$$

where $p_{\mathrm{T}, \ell \ell^{\prime}}$ and $m_{\ell \ell^{\prime}}$ are the transverse momentum and the mass of the di-lepton system, respectively, $\not_{\mathrm{T}}$ is the missing transverse momentum, and $\Delta \phi_{\ell \ell^{\prime}, \not_{\mathrm{T}}}$ is the difference in 
azimuth between $\mathbb{E}_{\mathrm{T}}$ and $p_{\mathrm{T}, \ell \ell^{\prime}}$. After a pre-selection $(\mathrm{P})$, additional cuts are applied that define a signal $(\mathrm{S})$ and a control $(\mathrm{C})$ region. The latter is exploited to normalise background simulations to data in the experimental analyses in each jet bin. In the ATLAS analysis, different cuts are applied in the 0 - and 1-jet bins. All cuts have been implemented in form of a RIVET [66] analysis.

\section{Acknowledgments}

We are grateful to A. Denner, S. Dittmaier and L. Hofer for providing us with the one-loop tensor-integral library CoLLIER. We thank T. Gehrmann for discussions and S. Kallweit for cross-checking four-lepton plus 0- and 1-jet NLO cross sections. The research of F. Cascioli, P. Maierhöfer and S. Pozzorini is supported by the SNSF. Stefan Höche was supported by the U.S. Department of Energy under Contract No. DE-AC02-76SF00515. Frank Siegert's work was supported by the German Research Foundation (DFG) via grant DI 784/2-1. We gratefully thank the bwGRiD project for computational resources. This research used resources of the National Energy Research Scientific Computing Center, which is supported by the Office of Science of the U.S. Department of Energy under Contract No. DE-AC02$05 \mathrm{CH} 11231$.

Open Access. This article is distributed under the terms of the Creative Commons Attribution License (CC-BY 4.0), which permits any use, distribution and reproduction in any medium, provided the original author(s) and source are credited.

\section{References}

[1] ATLAS collaboration, Observation of a new particle in the search for the standard model Higgs boson with the ATLAS detector at the LHC, Phys. Lett. B 716 (2012) 1 [arXiv: 1207.7214] [INSPIRE].

[2] CMS collaboration, Observation of a new boson at a mass of $125 \mathrm{GeV}$ with the CMS experiment at the LHC, Phys. Lett. B 716 (2012) 30 [arXiv:1207.7235] [INSPIRE].

[3] ATLAS collaboration, Measurements of the properties of the Higgs-like boson in the $W W^{*} \rightarrow \ell \ell \nu \nu$ decay channel with the ATLAS detector using $25 \mathrm{fb}^{-1}$ of proton-proton collision data, ATLAS-CONF-2013-030 (2013).

[4] CMS collaboration, Evidence for a particle decaying to $W^{+} W^{-}$in the fully leptonic final state in a standard model Higgs boson search in pp collisions at the LHC, CMS-PAS-HIG-13-003 (2013).

[5] J. Ohnemus, An order $\alpha_{s}$ calculation of hadronic $W^{-} W^{+}$production, Phys. Rev. D 44 (1991) 1403 [INSPIRE].

[6] S. Frixione, A next-to-leading order calculation of the cross-section for the production of $W^{+} W^{-}$pairs in hadronic collisions, Nucl. Phys. B 410 (1993) 280 [INSPIRE].

[7] J. Ohnemus, Hadronic $Z Z, W^{-} W^{+}$and $W^{ \pm} Z$ production with $Q C D$ corrections and leptonic decays, Phys. Rev. D 50 (1994) 1931 [hep-ph/9403331] [INSPIRE]. 
[8] L.J. Dixon, Z. Kunszt and A. Signer, Vector boson pair production in hadronic collisions at order $\alpha_{s}$ : lepton correlations and anomalous couplings, Phys. Rev. D 60 (1999) 114037 [hep-ph/9907305] [INSPIRE].

[9] J.M. Campbell and R.K. Ellis, An update on vector boson pair production at hadron colliders, Phys. Rev. D 60 (1999) 113006 [hep-ph/9905386] [INSPIRE].

[10] J.M. Campbell, R.K. Ellis and C. Williams, Vector boson pair production at the LHC, JHEP 07 (2011) 018 [arXiv: 1105.0020] [INSPIRE].

[11] S. Frixione and B.R. Webber, Matching NLO QCD computations and parton shower simulations, JHEP 06 (2002) 029 [hep-ph/0204244] [INSPIRE].

[12] S. Frixione, P. Nason and C. Oleari, Matching NLO QCD computations with parton shower simulations: the POWHEG method, JHEP 11 (2007) 070 [arXiv:0709.2092] [INSPIRE].

[13] T. Melia, P. Nason, R. Rontsch and G. Zanderighi, $W^{+} W^{-}, W Z$ and $Z Z$ production in the POWHEG BOX, JHEP 11 (2011) 078 [arXiv:1107.5051] [INSPIRE].

[14] R. Frederix et al., Four-lepton production at hadron colliders: aMC@NLO predictions with theoretical uncertainties, JHEP 02 (2012) 099 [arXiv: 1110.4738] [INSPIRE].

[15] J.M. Campbell, R.K. Ellis and G. Zanderighi, Next-to-leading order predictions for $W W+1$ jet distributions at the LHC, JHEP 12 (2007) 056 [arXiv:0710.1832] [INSPIRE].

[16] S. Dittmaier, S. Kallweit and P. Uwer, $N L O Q C D$ corrections to $W W+$ jet production at hadron colliders, Phys. Rev. Lett. 100 (2008) 062003 [arXiv: 0710.1577] [INSPIRE].

[17] S. Dittmaier, S. Kallweit and P. Uwer, $N L O Q C D$ corrections to $p p / p \bar{p} \rightarrow W W+j e t+X$ including leptonic W-boson decays, Nucl. Phys. B 826 (2010) 18 [arXiv:0908.4124] [INSPIRE].

[18] B. Jager, C. Oleari and D. Zeppenfeld, Next-to-leading order QCD corrections to $W^{+} W^{-}$ production via vector-boson fusion, JHEP 07 (2006) 015 [hep-ph/0603177] [INSPIRE].

[19] B. Jager and G. Zanderighi, Electroweak $W^{+} W^{-} j j$ prodution at $N L O$ in $Q C D$ matched with parton shower in the POWHEG-BOX, JHEP 04 (2013) 024 [arXiv: 1301.1695] [INSPIRE].

[20] T. Melia, K. Melnikov, R. Rontsch and G. Zanderighi, $N L O$ QCD corrections for $W^{+} W^{-}$ pair production in association with two jets at hadron colliders, Phys. Rev. D 83 (2011) 114043 [arXiv: 1104.2327] [INSPIRE].

[21] N. Greiner et al., NLO QCD corrections to the production of $W^{+} W^{-}$plus two jets at the LHC, Phys. Lett. B 713 (2012) 277 [arXiv:1202.6004] [INSPIRE].

[22] T. Melia, K. Melnikov, R. Rontsch and G. Zanderighi, Next-to-leading order QCD predictions for $W^{+} W^{+} j j$ production at the LHC, JHEP 12 (2010) 053 [arXiv:1007.5313] [INSPIRE].

[23] A. Denner, L. Hosekova and S. Kallweit, $N L O$ QCD corrections to $W^{+} W^{+} j j$ production in vector-boson fusion at the LHC, Phys. Rev. D 86 (2012) 114014 [arXiv:1209.2389] [INSPIRE].

[24] B. Jager and G. Zanderighi, NLO corrections to electroweak and QCD production of $W^{+} W^{+}$ plus two jets in the POWHEGBOX, JHEP 11 (2011) 055 [arXiv:1108.0864] [INSPIRE].

[25] F. Campanario, M. Kerner, L.D. Ninh and D. Zeppenfeld, $W Z$ production in association with two jets at NLO in QCD, Phys. Rev. Lett. 111 (2013) 052003 [arXiv:1305.1623] [INSPIRE].

[26] T. Binoth, M. Ciccolini, N. Kauer and M. Krämer, Gluon-induced WW background to Higgs boson searches at the LHC, JHEP 03 (2005) 065 [hep-ph/0503094] [INSPIRE]. 
[27] T. Binoth, M. Ciccolini, N. Kauer and M. Krämer, Gluon-induced W-boson pair production at the LHC, JHEP 12 (2006) 046 [hep-ph/0611170] [INSPIRE].

[28] J.M. Campbell, R.K. Ellis and C. Williams, Gluon-gluon contributions to $W^{+} W^{-}$production and Higgs interference effects, JHEP 10 (2011) 005 [arXiv: 1107.5569] [INSPIRE].

[29] T. Melia, K. Melnikov, R. Rontsch, M. Schulze and G. Zanderighi, Gluon fusion contribution to $W^{+} W^{-}+$jet production, JHEP 08 (2012) 115 [arXiv:1205.6987] [INSPIRE].

[30] N. Kauer and G. Passarino, Inadequacy of zero-width approximation for a light Higgs boson signal, JHEP 08 (2012) 116 [arXiv:1206.4803] [INSPIRE].

[31] LHC Higgs Cross Section Working Group collaboration, S. Heinemeyer et al., Handbook of LHC Higgs Cross Sections: 3. Higgs Properties, arXiv:1307.1347 [INSPIRE].

[32] T. Gleisberg et al., Event generation with SHERPA 1.1, JHEP 02 (2009) 007 [arXiv:0811.4622] [INSPIRE].

[33] F. Cascioli, P. Maierhofer and S. Pozzorini, Scattering amplitudes with open loops, Phys. Rev. Lett. 108 (2012) 111601 [arXiv:1111.5206] [INSPIRE].

[34] A. Denner, S. Dittmaier and L. Hofer, in preparation.

[35] A. Denner and S. Dittmaier, Reduction of one loop tensor five point integrals, Nucl. Phys. B 658 (2003) 175 [hep-ph/0212259] [INSPIRE].

[36] A. Denner and S. Dittmaier, Reduction schemes for one-loop tensor integrals, Nucl. Phys. B 734 (2006) 62 [hep-ph/0509141] [INSPIRE].

[37] A. Denner and S. Dittmaier, Scalar one-loop 4-point integrals, Nucl. Phys. B 844 (2011) 199 [arXiv: 1005.2076] [INSPIRE].

[38] S. Hoeche, F. Krauss, M. Schonherr and F. Siegert, A critical appraisal of NLO+PS matching methods, JHEP 09 (2012) 049 [arXiv:1111.1220] [INSPIRE].

[39] S. Hoeche, F. Krauss, M. Schonherr and F. Siegert, $W+n$-jet predictions at the Large Hadron Collider at next-to-leading order matched with a parton shower, Phys. Rev. Lett. 110 (2013) 052001 [arXiv:1201.5882] [INSPIRE].

[40] T. Gehrmann, S. Hoche, F. Krauss, M. Schonherr and F. Siegert, NLO QCD matrix elements + parton showers in $e^{+} e^{-} \rightarrow$ hadrons, JHEP 01 (2013) 144 [arXiv:1207.5031] [INSPIRE].

[41] S. Hoeche, F. Krauss, M. Schonherr and F. Siegert, QCD matrix elements + parton showers: The NLO case, JHEP 04 (2013) 027 [arXiv:1207.5030] [INSPIRE].

[42] A. Denner, S. Dittmaier, M. Roth and L. Wieders, Electroweak corrections to charged-current $e^{+} e^{-} \rightarrow 4$ fermion processes: technical details and further results, Nucl. Phys. B 724 (2005) 247 [Erratum ibid. B 854 (2012) 504] [hep-ph/0505042] [INSPIRE].

[43] A. Bredenstein, A. Denner, S. Dittmaier and S. Pozzorini, NLO QCD corrections to $p p \rightarrow t \bar{t} b \bar{b}+X$ at the LHC, Phys. Rev. Lett. 103 (2009) 012002 [arXiv:0905.0110] [INSPIRE].

[44] A. Bredenstein, A. Denner, S. Dittmaier and S. Pozzorini, NLO QCD corrections to top anti-top bottom anti-bottom production at the LHC: 2. full hadronic results, JHEP 03 (2010) 021 [arXiv: 1001.4006] [INSPIRE].

[45] A. Denner, S. Dittmaier, S. Kallweit and S. Pozzorini, NLO QCD corrections to WWbb production at hadron colliders, Phys. Rev. Lett. 106 (2011) 052001 [arXiv:1012.3975] [INSPIRE]. 
[46] A. Denner, S. Dittmaier, S. Kallweit and S. Pozzorini, NLO QCD corrections to off-shell top-antitop production with leptonic decays at hadron colliders, JHEP 10 (2012) 110 [arXiv: 1207.5018] [INSPIRE].

[47] A. van Hameren, Multi-gluon one-loop amplitudes using tensor integrals, JHEP 07 (2009) 088 [arXiv: 0905.1005] [INSPIRE].

[48] P. Draggiotis, M. Garzelli, C. Papadopoulos and R. Pittau, Feynman rules for the rational part of the QCD 1-loop amplitudes, JHEP 04 (2009) 072 [arXiv:0903.0356] [INSPIRE].

[49] G. Ossola, C.G. Papadopoulos and R. Pittau, Reducing full one-loop amplitudes to scalar integrals at the integrand level, Nucl. Phys. B 763 (2007) 147 [hep-ph/0609007] [INSPIRE].

[50] S. Catani, Y.L. Dokshitzer, M. Olsson, G. Turnock and B. Webber, New clustering algorithm for multi-jet cross-sections in $e^{+} e^{-}$annihilation, Phys. Lett. B 269 (1991) 432 [INSPIRE].

[51] S. Catani, B. Webber and G. Marchesini, $Q C D$ coherent branching and semiinclusive processes at large $x$, Nucl. Phys. B 349 (1991) 635 [InSPIRE].

[52] I.W. Stewart and F.J. Tackmann, Theory uncertainties for Higgs and other searches using jet bins, Phys. Rev. D 85 (2012) 034011 [arXiv:1107.2117] [InSPIRE].

[53] S. Catani and M. Seymour, A general algorithm for calculating jet cross-sections in $N L O$ QCD, Nucl. Phys. B 485 (1997) 291 [Erratum ibid. B 510 (1998) 503] [hep-ph/9605323] [INSPIRE].

[54] S. Schumann and F. Krauss, A parton shower algorithm based on Catani-Seymour dipole factorisation, JHEP 03 (2008) 038 [arXiv:0709.1027] [INSPIRE].

[55] S. Hoeche, S. Schumann and F. Siegert, Hard photon production and matrix-element parton-shower merging, Phys. Rev. D 81 (2010) 034026 [arXiv:0912.3501] [INSPIRE].

[56] S. Hoeche, F. Krauss, S. Schumann and F. Siegert, QCD matrix elements and truncated showers, JHEP 05 (2009) 053 [arXiv: 0903.1219] [INSPIRE].

[57] S. Catani, F. Krauss, R. Kuhn and B. Webber, QCD matrix elements + parton showers, JHEP 11 (2001) 063 [hep-ph/0109231] [INSPIRE].

[58] P. Agrawal and A. Shivaji, Di-vector boson + jet production via gluon fusion at hadron colliders, Phys. Rev. D 86 (2012) 073013 [arXiv:1207.2927] [INSPIRE].

[59] H.-L. Lai et al., New parton distributions for collider physics, Phys. Rev. D 82 (2010) 074024 [arXiv: 1007.2241] [INSPIRE].

[60] J.M. Campbell and R. Ellis, MCFM for the Tevatron and the LHC, Nucl. Phys. Proc. Suppl. 205-206 (2010) 10 [arXiv:1007.3492] [INSPIRE].

[61] F. Krauss, R. Kuhn and G. Soff, $A M E G I C++$ 1.0: a matrix element generator in $\mathrm{C}++$, JHEP 02 (2002) 044 [hep-ph/0109036] [INSPIRE].

[62] T. Gleisberg and S. Hoeche, Comix, a new matrix element generator, JHEP 12 (2008) 039 [arXiv:0808.3674] [INSPIRE].

[63] T. Gleisberg and F. Krauss, Automating dipole subtraction for QCD NLO calculations, Eur. Phys. J. C 53 (2008) 501 [arXiv:0709.2881] [INSPIRE].

[64] L. Lönnblad and S. Prestel, Unitarising matrix element + parton shower merging, JHEP 02 (2013) 094 [arXiv:1211.4827] [INSPIRE]. 
[65] L. Lönnblad and S. Prestel, Merging multi-leg NLO matrix elements with parton showers, JHEP 03 (2013) 166 [arXiv: 1211.7278] [INSPIRE].

[66] A. Buckley et al., Rivet user manual, Comput. Phys. Commun. 184 (2013) 2803 [arXiv: 1003.0694] [INSPIRE].

[67] M. Cacciari, G.P. Salam and G. Soyez, The anti- $k_{t}$ jet clustering algorithm, JHEP 04 (2008) 063 [arXiv:0802.1189] [INSPIRE]. 